\title{
APPLICATION OF THE CONTINUUM REPLICA METHODOLOGY FOR THE DESCRIPTION OF STRUCTURE AND THERMODYNAMICS OF QUENCHED-ANNEALED FLUID SYSTEMS
}

\author{
O. Pizio ${ }^{1}$, S. Sokolowski ${ }^{2}$ \\ ${ }^{1}$ Instituto de Química de la UNAM, Coyoacán 04510, México D. F. \\ ${ }^{2}$ Department of Chemistry Marie Curie-Sklodowska University, Lublin, 200-31, Poland
}

(Received November 17, 1997)

\begin{abstract}
In this review we have considered a problem of the description of quenched-annealed fluids in the framework of the liquid-state replica methodology. A statistical mechanical fundamentals of the theory are considered. Interesting developments and important results for the structure, thermodynamics, and particularly, for phase transitions in these systems, already available in the literature, are discussed. Our emphasis also is focused on the original contributions of the authors. We discuss results for two-dimensional partly quenched models, for inhomogeneous systems and chemically associating fluids in disordered porous environment. Charged annealed fluids adsorbed in charged disordered matrices are investigated finally. Mostly we consider the applications of the replica type integral equations for all the systems mentioned above. However, a computer simulation approach also is discussed and some original simulation data are given. In conclusion, we outline the issues unsolved so far and discuss areas which seem promising for future developments.

Key words: replica, adsorption, microporous media, integral equations, computer simulation, structure, thermodynamics, phase transitions.
\end{abstract}

PACS number(s): 61.20.Gy, 61.43.Bn, 64.70.Fx, 68.45.-v, 81.05.Rm, 82.30.Nr, 82.60.Lf, 82.70.Gg

\section{INTRODUCTION}

Very recently the scientific interests of our research group have turned on to the studies of quenchedannealed fluids. One can observe augmenting activity in this area of the leading theoretical laboratories, which have involved the groups of G. Stell and J. Given, of E. D. Glandt and W. G. Madden, of M. L. Rosinberg and E. Kierlik, and of P. Monson and R.D. Kaminsky, among the others.

To our best knowledge, there has not been a comprehensive review of the theoretical studies of quenchedannealed fluid systems. Therefore, our intention in this work is to cover, at least partially, the existing vacuum. Evidently, it is impossible to discuss the state of art in every detail in one paper. We will omit, for example, the discussion of the fundamentals of the replica method for lattice systems referring the reader to the monograph [1]. However, it is a real pleasure for us to present our contribution to the Journal of Physical Studies, because it is closely related to the area of interests of I. A. Vakarchuk and Yu. K. Rudavskii, who both together with their coworkers, have contributed much into the extension and the application of the replica method for liquid magnetic systems $[2-5]$.

The structure of this review is as follows. First we make a brief introduction (section I) referring to the most important developments as well as interesting experimental observations. In section II we present fundamental relations of the liquid-state replica methodology. Those include the definitions for the partition function and averaged grand thermodynamic potential, for the fluctuations in the system and the correlation functions. Our derivation has been developed in canonical and grand canonical ensemble. In the second part of this section the replica Ornstein-Zernike (OZ) equations and closure approximations are discussed. We terminate the presentation of theoretical background with derivation of thermodynamic properties of partly quenched systems.

In section III our presentation is focused on the most important results obtained by different authors in the method of integral equations and in simulations for simple fluids. Besides, we discuss our original results obtained very recently for two-dimensional and threedimensional models of quenched-annealed fluids. Everywhere, if possible, we make comparisons with computer simulation results.

Section IV is dedicated to the development of the theory of inhomogeneous partly quenched systems. The theory involves the inhomogeneous, or the second order, replica OZ equations and the Born-Green-Yvon equation for the density profile of adsorbed fluid in disordered media. Some computer simulation results also are given.

Then, in section $\mathrm{V}$, we turn our attention to the problem of chemically associating systems adsorbed in disordered porous media. We present associative extension of the replica OZ equations, discuss a set of approximations for them and the derivation of thermodynamic properties. Some original results also are given. All previous developments concern fluids without electrostatic forces. However, the problem of charged fluids adsorbed in charged disordered matrices is of much interest for basic and applied research.

Therefore, we proceed in section VI with the theory of quenched-annealed charged systems which very recently have become the subject of our research. Peculiarities of the screening of Coulomb interactions in ad- 
sorbed charged fluids restricted to charged obstacles is discussed. In conclusion, we present a summarizing discussion and outline possible future studies that are of interest in our opinion this area.

We would like, however, to start with a clear description of the subject of our work. We are interested in quenched-annealed, or partly quenched, systems, i.e. in those in which some degrees of freedom are quenched (fixed) whereas the others are allowed to equilibrate. This equilibration, evidently, occurs in the presence of a subsystem of quenched variables. The ensemble averages corresponding to the expectation values of physical quantities in fact represent double averages. Thermal averaging must be performed with the quenched variables kept fixed, and then averaging over a spectrum of values of the quenched degrees of freedom must be done.

Continuum systems with quenched disorder include, for example, porous and sintered porous materials, gels, amorphous substances, glasses, engineering composites, etc. Related to the subject of our presentation we would like to recall that porous media include silica gels, polymer organic gels and porous glasses, such as Vycor. To have a better idea about the physical structure of porous media we would deal with, it is worth mentioning that Vycor has a porosity about $30 \%$ and consists of interconnecting pores of the radius $\approx 30 \AA$. In contrast to that structure, gels may be thought as a network of interconnecting strands and are characterized by very high porosities, in the range of $90-98 \%$. Therefore, the behaviour of adsorbed fluids in these two types of materials may differ much. A more detailed description of porous systems can be found in [6]. Experimental studies of partly quenched systems have discovered their unusual thermodynamic properties. It was shown that the liquidvapour transition and liquid-liquid separation are quite different in annealed and partly quenched systems [7-9]. A remarkable narrowing of the liquid-vapour coexistence curve, in comparison with that for the bulk, has been observed for ${ }^{4} \mathrm{He}$ in aerogel [8]. Besides, a phase separation in a mixture at a temperature sufficiently below the critical temperature of the bulk mixture has been found [9]. Interesting recent experimental observations concerning the formation of liquid crystalline phases in disordered porous media have been discussed by F. Aliev [10].

Dynamic aspects of the behaviour of colloidal systems in porous media also have been studied [11-13]. In particular, G. Viramontes-Gamboa et al. [11, 12], extending the experimental and simulative research in colloids in the laboratory of M. Medina-Noyola, have performed Brownian dynamics simulation to investigate tracer diffusion in a colloidal suspension under the conditions that one of the colloidal species is frozen. The works cited above may be discussed in further detail, however, our focus in this review is the theory, rather than the experiment.

Theoretical studies of quenched-annealed systems have been initiated with success by Madden and Glandt $[14,15]$; these authors have presented exact Mayer cluster expansions for the correlation functions for the case when the matrix, i.e. quenched species, subsystem is gen- erated by a quench from an equilibrium distribution, as well as for the case of arbitrary distribution of obstacles. However, their integral equations for the correlation functions appeared to be only approximate; it has been demonstrated explicitly by Given and Stell [16]. By performing a detailed analysis of the cluster series of Madden and Glandt, Given and Stell have derived a correct set of equations and called them the replica Ornstein-Zernike (ROZ) equations [16-18]. The closure relations have been proposed for the ROZ equations and tested by comparison with computer simulation data [19, 20]. However, thermodynamic properties of quenchedannealed systems are much more difficult to obtain both from the theory and from simulations. This problem has been addressed in [21-26], but still lacks complete solution. Majority of aforementioned studies, in their practical part, have restricted to adsorbed fluids with only repulsive interparticle forces, such that the question of liquid-vapor transition had left out of question. Recently, a mean field theory has been applied for fluids with square-well attraction [23]. On the other hand, Kierlik et al. [26] have used optimized cluster expansions to investigate the phase diagram of Lennard-.Jones fluids adsorbed in a hard sphere disordered matrix. In spite of excellent theoretical analysis of the critical behaviour of a fluid in a disordered porous matrix of Pitard et al. [27], much room remains for improvement of the theory for the explanation of a specific shape liquid-vapor coexistence curve and of a liquid-liquid equilibrium. Those have been investigated by using grand canonical and Gibbs ensemble Monte Carlo simulations for sufficiently simple models (see Refs. [28] and [29], respectively), such that the theory must pass through severe tests. Actually the application of Gibbs ensemble methodology, as well as of isobaric ensemble, encounters very serious difficulties for other models than that studied in [29]. In that work, the volume changes of the simulative box have been unnecessary due to a special symmetry of the model.

There have been other promising lines along which the theory of quenched-annealed systems have progressed recently. One of them, worth to discuss in more detail, is the adsorption of fluids in the inhomogeneous, i.e. geometrically restricted, quenched media [30-32]. In this area one encounters severe methodological and technical difficulties. At the moment, a set of results has been obtained at the level of a hard sphere type models adsorbed in a slit-like pores with quenched distribution of a hard sphere obstacles (Duda et al. [33] have made progress in simulations of matrix-filled cylindrical pores very recently. However, the problem of phase transitions remained out of question so far). The models and methodology available at present seem promising for the investigations of phase transformations in nonuniform partly quenched systems with more sophisticated interactions.

Sophistication of interactions for another purpose, has been attempted in a series of publications presented by our group, in particular. Namely, the focus has been directed on complex fluid behaviour in a quenched environment. Vega and Padilla have considered chain fluids in their simulative study [34]. On the other hand, the phe- 
nomena of chemical association (chemical reactions) in disordered porous materials have become our main concern. It is an interesting subject both from practical, see eg. $[35,36]$, and from theoretical point of view. The theory developed by Wertheim $[37,38]$ for directional chemical association, and generalized by Kalyuzhnyi and Stell [39] for spherically symmetric finite-range forces (both for the bulk fluids), provides a convenient basis to investigate chemically associating quenched-annealed systems. This extension of associating theory has been pioneered in $[40,41]$ and later followed by several applications [4248]. We present below these important developments in the framework of the associative ROZ equations in more detail.

Our final focus in this review is on charged quenched-annealed fluid systems. Very recently Bratko, Chakraborty and Chandler have addressed this problem in [49-51]. A set of grand canonical computer simulation results for infinitely diluted adsorbed fluid has been presented and an attempt to describe them at the level of Debye-Hückel type theory of electrolyte solutions has been undertaken [50]. On the other hand, we have consistently considered the problem using the ROZ equations for point ions and then for nonpoint charged particles $[52,53]$. The former case has been solved by us to perform renormalization of the ROZ equations in view of a long-range Coulomb interactions. However, interesting observations for the screening potentials of point ions in an environment of fixed point charges distribution have been obtained.

The theory of quenched-annealed fluids is rapidly developing. In this review we have attempted to present some of the problems already solved and to discuss only some of the issues that need further study. undoubtedly there remains much room for the theoretical developments. On the other hand accumulation of the theoretical and simulative results is required for further progress. Of particular importance are the issues related with phase transitions in these systems. The studies of the models with more sophisticated interactions than those involved at present would be of much interest too.

\section{REVIEW OF THE THEORETICAL BACKGROUND}

Let us consider the simplest model of quenchedannealed system which consists of particles belonging to two species: species 0 is quenched (matrix) and species 1 annealed, i.e. allowed to equilibrate between themselves in the presence of 0 particles. We assume that the subsystem composed of 0 particles has been a usual fluid (one can characterize it either by the density $\rho^{0}$, or by the value of the chemical potential $\mu^{0}$ ) before quench. The interparticle interaction $u_{00}(r)$ does not need to be specified for the moment. Just it is assumed that the fluid with interaction $u_{00}(r)$ has reached an equilibrium at certain temperature $T_{0}$, and then the fluid has been quenched at this temperature. Thus the distribution of species 0 is any one from a set of configurations corresponding to canonical or grand canonical ensemble). We denote the interactions between annealed particles as $u_{11}(r)$, and the "cross" fluid-matrix interactions as $u_{10}(r)$.

We introduce the notation $\mathbf{0}$ and $\mathbf{1}$ for the vector of coordinates of the quenched and annealed particles, respectively. For the sake of methodologically clear presentation we restrict ourselves to the canonical ensemble for the moment. The Helmholtz free energy of the partly quenched system in question is given by $[16-18,21]$

$$
\begin{aligned}
-\beta A_{P Q} & =\left\langle\ln Z_{1}\right\rangle_{0} \\
& =Z_{0}^{-1} \int d 0 \exp \left(-\beta_{0} H_{00}\right) \ln Z_{1}
\end{aligned}
$$

where $\beta_{0}=1 / k T_{0}$,

$$
\begin{gathered}
Z_{1}=\int d \mathbf{1} \exp \left[-\beta\left(H_{10}+H_{11}\right)\right] \\
Z_{0}=\int d \mathbf{0} \exp \left(-\beta_{0} H_{00}\right)
\end{gathered}
$$

are usual expressions for the partition sums, $H_{i j}$ are the Hamiltonians consisting of relevant pairwise interactions. It is important to mention that the temperature of observation of annealed fluid $T(\beta=1 / k T)$ does not necessarily coincide with the temperature $T_{0}$ at which matrix has been quenched.

The logarithm of the partition sum of annealed species under the sign of integration in eq. (1) makes the analytical treatment difficult. Therefore the replica method, see, e.g. [1], is used, i.e. the following identity is exploited

$$
\ln Z=\lim _{s \rightarrow 0}\left(Z^{s}-1\right) /\left.s \equiv \frac{d}{d s}\right|_{s \rightarrow 0} Z^{s}
$$

where in eq. (3) $\lim _{s \rightarrow 0}(d / d s)$ has been denoted as $\left.\frac{d}{d s}\right|_{s \rightarrow 0}$, similar notation will be used below for the sake of brevity. The free energy of the system given by eq. (1) can be written then in the 'replicated' form

$$
\begin{aligned}
& -\beta A_{P Q}=\left.Z_{0}^{-1} \frac{d}{d s}\right|_{s \rightarrow 0} \int d \mathbf{0} \\
& \times \exp \left(-\beta_{0} H_{00}\right)(d \mathbf{1}) \exp \left\{-\beta \sum_{1 \leq s}\left(H_{10}^{(m)}+H_{11}^{(m)}\right)\right\},
\end{aligned}
$$

where the variables describing species $\mathbf{1}$ appear in $s$ copies or replicas according to the representation of the logarithm in form of eq. (3). Our notation $(d \mathbf{1})$ just indicates that integration is performed over $s$ sets of variables for annealed particles coordinates. The form provided by eq. (4) is much more convenient to apply, because it represents a limit, $s \rightarrow 0$, of the partition sum for an replicated system by which we mean an equilibrium system that is described by the following Hamiltonian

$$
\mathcal{H}=\sum_{i<j} u_{00}(i j)+\sum_{1 \leq m \leq s} \sum_{i<j} u_{10}^{m}(i j)
$$




$$
+\sum_{1 \leq m \leq s 1 \leq n \leq s i<j} \sum_{11} u_{11}^{m n}(i j)
$$

Here the superscripts serve as replica indices, they attribute the coordinates of particles to a given replica but do not alter interactions in the system

$$
\begin{gathered}
u_{10}^{m}(i j) \equiv u_{10}(i j), \\
u_{11}^{m n}(i j) \equiv \delta_{m n} u_{11}(i j),
\end{gathered}
$$

where $\delta_{m n}$ is the Kronecker symbol. However, the particles belonging to different replicas do not interact. As a result of the procedure in above the quenched-annealed system has been 'transformed' into an equilibrium system with nonadditive interactions which can be characterized by the partition sum $Z_{\text {rep }}$. It is worth mentioning that the $s \rightarrow 0$ limit of the partition function of the replicated system yields $Z_{0}$. Therefore equation (4) can be rewritten in the form

$$
-\beta A_{P Q}=\left.\frac{d}{d s}\right|_{s \rightarrow 0} \ln Z_{r e p}=\left.\frac{d}{d s}\right|_{s \rightarrow 0}\left(-\beta A_{r e p}(s)\right),
$$

which establishes the relation between the free energy of the original partly quenched system and its replicated counterpart [21]. Usually one does not need to bother with the fluctuations in the number of matrix particles. The experiment is performed to investigate adsorption of a fluid in a matrix (at average density $\rho^{0}$ ), i.e. in contact with a reservoir that fixes the chemical potential [21].

However, if one focuses on the adsorption of a fluid in heterogenous matrices [30-32] and/or on the fluctuations in an adsorbed fluid, it is inevitable to perform the developments similar to those in above in the grand canonical ensemble. Moreover this derivation is of importance for the formulation of the virial route to thermodynamics of partially quenched systems. We include only a few basic relations of this approach for better presentation.

By definition, an observable property of a partly quenched system, $f_{P Q}$, in the grand canonical ensemble is obtained as

$$
\begin{aligned}
& f_{P Q}=\langle f(\mathbf{0})\rangle_{0}=\left(1 / \Xi_{0}\right) \sum_{0 \leq N_{0} \leq \infty}\left(1 / N_{0} !\right) z_{0}^{N_{0}} \\
& \times \int d 0 f\left(N_{0}, \mathbf{0}\right) \exp \left(-\beta_{0} H_{00}\right),
\end{aligned}
$$

where $\beta_{0}=1 / k T_{0}$, the angular brackets $\langle\ldots\rangle_{0}$ denote an average over the quenched degrees of freedom, i.e. over the coordinates of matrix species; $N_{0}, z_{0}$ and $H_{00}$ are the average number, the activity of the matrix particles, and the Hamiltonian of the matrix system, respectively. The grand potential of this system is

$$
\Xi_{0}=\sum_{0 \leq N_{0} \leq \infty}\left(1 / N_{0} !\right) z_{0}^{N_{0}} \int d \mathbf{0} \exp \left(-\beta_{0} H_{00}\right)
$$

According to eq. (8) the pressure in terms of the grand canonical potential per unit volume is

$$
\beta V P^{P Q} \equiv \beta V\langle P(\mathbf{0})\rangle_{0}=\left\langle\ln \Xi_{1}(\mathbf{0})\right\rangle_{0} .
$$

Using then the replica representation, one can easily obtain

$$
\beta V P^{P Q}=\left.\frac{d}{d s}\right|_{s \rightarrow 0} \ln \Xi^{r e p}=\left.\frac{d}{d s}\right|_{s \rightarrow 0} \beta V P^{r e p} .
$$

In spite of simplicity and visual similarity of this equation and of eq. (7), we would like to note that eq. (11) leads to a nontrivial thermodynamics of a partially quenched system in terms of the correlation functions, see e.g. [24] for detailed discussion. Evidently. the principal route for $P^{P Q}$ and to the virial theorem is to exploit thermodynamics of the replicated system. However, a special care must be taken because the $V$ and $s$ derivatives do not commute. Moreover, the presence of two different temperatures requires attention in taking temperature derivatives, setting those temperatures equal, if appropriate, only at the end of the calculations.

To define the correlation functions of partly quenched systems it is expedient to consider fluctuations. There are two types of fluctuations: thermal fluctuations for a given configuration of matrix species, and fluctuations induced by disorder. We characterize the average over disorder of thermal fluctuations by the variance

$$
\begin{aligned}
\sigma_{\text {therm }}^{2} & =\left\langle\left\langle N_{1}\left(\mathbf{q}^{N_{0}}\right)^{2}\right\rangle_{t h}\right\rangle_{d} \\
& -\left\langle\left\langle N_{1}\left(\mathbf{q}^{N_{0}}\right)\right\rangle_{t h}^{2}\right\rangle_{d},
\end{aligned}
$$

and the fluctuations of the thermal average induced by disorder by

$$
\begin{aligned}
\sigma_{\text {disord }}^{2} & =\left\langle\left\langle N_{1}\left(\mathbf{q}^{N_{0}}\right)\right\rangle_{t h}^{2}\right\rangle_{d} \\
& -\left\langle\left\langle N_{1}\left(\mathbf{q}^{N_{0}}\right)\right\rangle_{t h}\right\rangle_{d}^{2},
\end{aligned}
$$

where $\langle\ldots\rangle_{t h}$ and $\langle\ldots\rangle_{d}$ denote thermal and disorder averaging, respectively. It is expected that the average density of fluid particles in a matrix $N_{1}\left(\mathbf{q}^{N_{0}}\right) / V$ to be a self-averaging quantity, i.e. $\sigma_{\text {disord }} / N_{1} \rightarrow 0$ in the thermodynamic limit.

In close relation with the fluctuations one may introduce the correlation functions. The pair density distribution function for fluid particles $\rho^{11}\left(\mathbf{r}_{1}, \mathbf{r}_{2}\right)$ is defined as the average over all realizations of the matrix structure of the function $\rho^{11}\left(\mathbf{r}_{1}, \mathbf{r}_{2} ; \mathbf{q}^{N_{0}}\right)$. One can define then the pair correlation function in a usual manner

$$
\begin{aligned}
\rho^{1}\left(\mathbf{r}_{1}\right) \rho^{1}\left(\mathbf{r}_{2}\right) h^{11}\left(\mathbf{r}_{1}, \mathbf{r}_{2}\right) & =\rho^{11}\left(\mathbf{r}_{1}, \mathbf{r}_{2}\right) \\
& -\rho^{1}\left(\mathbf{r}_{1}\right) \rho^{1}\left(\mathbf{r}_{2}\right),
\end{aligned}
$$


in terms of averaged pair distribution function and the density profiles. The matrix is statistically homogeneous, therefore the fluid density is constant throughout the matrix, $\rho^{1}(\mathbf{r})=\rho^{1}$, where $\rho^{1}=N_{1} / V=\left\langle\left\langle N_{1}\left(\mathbf{q}^{N_{0}}\right)\right\rangle_{t h}\right\rangle_{d} / V$. The pair correlation function then depends only on the distance between particles, i.e. $h^{11}\left(\mathbf{r}_{1}, \mathbf{r}_{2}\right)=h\left(\left|\mathbf{r}_{12}\right|\right)$.

On the other hand, the connected pair distribution function for a given matrix configuration $\rho^{11, c}\left(\mathbf{r}_{1}, \mathbf{r}_{2} ; \mathbf{q}^{N_{0}}\right)$,

$$
\begin{aligned}
\rho^{11, c}\left(\mathbf{r}_{1}, \mathbf{r}_{2} ; \mathbf{q}^{N_{0}}\right) & =\rho^{11}\left(\mathbf{r}_{1}, \mathbf{r}_{2} ; \mathbf{q}^{N_{0}}\right) \\
& -\rho^{1}\left(\mathbf{r}_{1} ; \mathbf{q}^{N_{0}}\right) \rho^{1}\left(\mathbf{r}_{2} ; \mathbf{q}^{N_{0}}\right)
\end{aligned}
$$

generates two additional correlation functions (those are called the connected and blocking correlation functions)

$$
\begin{aligned}
& \left(\rho^{1}\right)^{2} h^{11, c}\left(\mathbf{r}_{1}, \mathbf{r}_{2}\right)=\left\langle\rho^{11, c}\left(\mathbf{r}_{1}, \mathbf{r}_{2} ; \mathbf{q}^{N_{0}}\right)\right\rangle_{d} \\
= & \rho^{11}\left(\mathbf{r}_{1}, \mathbf{r}_{2}\right)-\left\langle\rho^{1}\left(\mathbf{r}_{1} ; \mathbf{q}^{N_{0}}\right) \rho^{1}\left(\mathbf{r}_{2} ; \mathbf{q}^{N_{0}}\right)\right\rangle_{d}
\end{aligned}
$$

and

$$
\begin{aligned}
\left(\rho^{1}\right)^{2} h^{11, b}\left(\mathbf{r}_{1}, \mathbf{r}_{2}\right) & =\left\langle\rho^{1}\left(\mathbf{r}_{1} ; \mathbf{q}^{N_{0}}\right) \rho^{1}\left(\mathbf{r}_{2} ; \mathbf{q}^{N_{0}}\right)\right\rangle_{d} \\
& -\left(\rho^{1}\right)^{2}
\end{aligned}
$$

such that $h^{11}\left(r_{12}\right)=h^{11, c}\left(r_{12}\right)+h^{11, b}\left(r_{12}\right)$. The isothermal compressibility of adsorbed fluid, $\chi_{1}$, has been shown in [21], is related to the averaged thermal average as follows

$$
\rho_{1} k T \chi_{1}=\sigma_{\text {therm }}^{2} / N_{1}
$$

The one-particle density, $\rho^{1}\left(\mathbf{r}_{1} ; \mathbf{q}^{N_{0}}\right)$, and the pair density, $\rho^{11}\left(\mathbf{r}_{1}, \mathbf{r}_{2} ; \mathbf{q}^{N_{0}}\right)$, are normalized in the grand canonical ensemble, as usual,

$$
\begin{aligned}
& \int d \mathbf{r}_{1} \rho^{1}\left(\mathbf{r}_{1} ; \mathbf{q}^{N_{0}}\right)=\left\langle N_{1}\left(\mathbf{q}^{N_{0}}\right)\right\rangle_{t h} \\
& \int d \mathbf{r}_{1} d \mathbf{r}_{2} \rho^{11}\left(\mathbf{r}_{1}, \mathbf{r}_{2} ; \mathbf{q}^{N_{0}}\right)=\left\langle N_{1}\left(\mathbf{q}^{N_{0}}\right)^{2}\right\rangle_{t h} \\
& -\left\langle N_{1}\left(\mathbf{q}^{N_{0}}\right)\right\rangle_{t h}
\end{aligned}
$$

Then, performing a disorder average in eq. (19), and using eq. (18) we can obtain the following two relations for the connected and blocking correlation functions

$$
\begin{gathered}
\rho^{1} k T \chi_{1}=\sigma_{\text {therm }}^{2} / N_{1}=1+\rho^{1} \int d \mathbf{r}_{12} h^{11, c}\left(r_{12}\right) \\
\rho^{1} \int d \mathbf{r}_{12} h^{11, b}\left(r_{12}\right)=\sigma_{\text {disord }}^{2} / N_{1}
\end{gathered}
$$

The former of these two relations is the compressibility equation for the fluid in the matrix. Its worth mentioning that this expression is the only practical route, for the moment, to calculate the fluid pressure for the systems in question.

We proceed with cluster series which yield the integral equations. Evidently the correlation functions presented above can be defined by their diagrammatic expansions. In particular, the blocking correlation function, $h^{11, b}\left(r_{12}\right)$ is the subset of graphs of $h^{11}\left(r_{12}\right)$ such that all paths between the fluid root points 1 and 2 pass through at least one matrix $\rho^{0}$ - field point. The direct correlation function $c^{11}$ is the sum of all graphs in $h^{11}$ with no nodal points. The cluster expansions for the correlation functions have been first obtained and analyzed in detail by Madden and Glandt [14, 15]. However, the exact equations for the correlation functions, those have been called the replica Ornstein-Zernike (ROZ) equations have been derived by Given and Stell [16-18]. These equations have the following form:

$$
h^{00}-c^{00}=\rho^{0} c^{00} \otimes h^{00},
$$

for the matrix-matrix correlations,

$$
h^{10}-c^{10}=\rho^{0} c^{10} \otimes h^{00}+\rho^{1} c^{11, c} \otimes h^{10},
$$

for the fluid-matrix correlations, and finally two equations for the fluid-fluid correlations

$$
\begin{aligned}
& h^{11}-c^{11}=\rho^{0} c^{10} \otimes h^{01}+\rho^{1} c^{11, c} \otimes h^{11} \\
& +\rho^{1} c^{11, b} \otimes h^{11, c}, \\
& h^{11, c}-c^{11, c}=\rho^{1} c^{11, c} \otimes h^{11, c} .
\end{aligned}
$$

In all equations above we have omitted the dependencies on $r$, the symbol $\otimes$ denotes convolution in $r$-space. The symmetry of the correlation functions implies that $h^{10}=h^{01}, c^{10}=c^{01}$. Similarly to the total pair correlation functions above, the direct correlation function of fluid particles also has been presented as a sum of the connected and blocking terms, $c^{11}=c^{11, c}+c^{11, b}$. It is important to mention, that the equation for the matrix structure decouples from the equations for the fluid-matrix and fluid-fluid structure. This appears because both these correlations do not have influence on the matrix structure.

To solve the replica OZ equations, they must be completed by the closure relations. Several closures have been tested against computer simulations for various models of fluids adsorbed in disordered porous media. In particular, the standard Percus-Yevick (PY) and the hypernetted chain approximations have been applied. The equation (21) for the matrix correlations can be solved using any approximation. However, it has been shown by Given and Stell [16] that the PY closure for the fluidfluid correlations is not completely consistent with the ROZ equation, the blocking effects of the matrix structure are neglected in this approximation, i.e. $c^{11, b}(r)=0$. Therefore the PY approximation may yield (and really it happens for partly quenched models with hard core re- 
pulsion between species) sufficiently accurate results due to mutual cancellation of inaccuracies in the description of fluid-matrix and fluid-fluid correlations on the background of neglected effect of the blocking direct correlation function. Lomba et al. [19] have proposed replica extensions for the reference $\mathrm{PY}$ and reference $\mathrm{HNC}$ closures, but have tested only the reference PY one, for a simple model of adsorbed fluid in a hard sphere disordered matrix. Meroni et al. [20] have demonstrated that the inclusion of the bridge diagrams contribution in the parametrized form improves the accuracy of the common hypernetted chain closure. However, these authors in their evaluation of the bridge diagrams term have used a set of effective diameters which is difficult to justify in the limit $s \rightarrow 0$ for the number of replicas. We will discuss adequacy of closures in more detail, while presenting the results for particular models.

Finally, in this part of the work we would like to discuss to some extent practical tools to obtain thermodynamic properties of adsorbed fluids. We have mentioned in above, that the compressibility equation is the only simple recipe, for the moment, to obtain the fluid compressibility and pressure. The reason is that the virial equation is difficult to implement, it has not been tested for partly quenched systems. Still for the sake of completeness we present the virial equation in the form [21]

$$
\begin{aligned}
\beta P^{1} & -\left.\rho^{0}\left[\partial\left(\beta P^{1}\right) / \partial \rho^{0}\right]\right|_{\mu^{1}}=\rho^{1} \\
& +\frac{2 \pi}{3}\left(\rho^{0}\right)^{2} \sigma_{0}^{3} \lim _{s \rightarrow 0}\left[d g_{r e p}^{00}\left(\sigma_{0} ; s\right) / d s\right] \\
& +\frac{2 \pi}{3}\left(\rho^{1}\right)^{2} \sigma_{1}^{3} g^{11}\left(\sigma_{1}\right)+\frac{4 \pi}{3} \rho^{0} \rho^{1} \sigma_{01}^{3} g^{01}\left(\sigma_{01}\right),
\end{aligned}
$$

where $\sigma_{01}=0.5\left(\sigma_{0}+\sigma_{1}\right), \sigma_{i i}=\sigma_{i}$, and $g^{i j}\left(\sigma_{i j}\right)$ are the contact values of the corresponding distribution functions. The subscript rep corresponds to the replicated system with $s$ replicas. We must note that the second term in the lhs and the first term in the rhs of this equation do not have exact representation. If one neglects the contribution of the order $\rho^{0}\left(\rho^{1}\right)^{2}$ in both of these terms, then [21]

$$
\begin{aligned}
& \lim _{s \rightarrow 0}\left[d g_{r e p}^{00}\left(\sigma_{0} ; s\right) / d s\right]=g_{r e p}^{00}(r ; s)\left[W_{r e p}^{00}(r ; s=1)\right. \\
& \left.-W_{r e p}^{00}(r ; s=0)\right], \\
& W_{r e p}^{00}(r ; s)=\ln g_{r e p}^{00}(r ; s)+\beta U_{00}(r), \\
& -\left.\left[\partial\left(\beta P^{1}\right) / \partial \rho^{0}\right]\right|_{\mu^{1}}=\beta \mu_{r e p}^{0}(s=1)-\beta \mu_{r e p}^{0}(s=0) .
\end{aligned}
$$

where $W_{r e p}^{00}(r ; s)$ is the excess potential of mean force for the replicated system. To use this virial equation one needs to consider equilibrium ensembles made of either matrix particles or a mixture of matrix and fluid particles, in addition to terms involving the pair distribution functions.

The equilibrium between the bulk fluid and fluid ad- sorbed in disordered porous media must be discussed at fixed chemical potential. Evaluation of the chemical potential for adsorbed fluid is a key issue for the adsorption isotherms, in studying phase diagram of adsorbed fluid, and for performing comparisons of the structure of a fluid in different media. At present, one of popular tools to obtain the chemical potentials have become an approach proposed by Ford and Glandt in [22]. From the detailed analysis of the cluster expansions these authors have concluded that the derivative of the excess chemical potential with respect to the fluid density equals to the connected part of the fluid-fluid direct correlation function (dcf). Then, it follows that the chemical potential of a fluid adsorbed in a disordered matrix, $\mu^{1}\left(\rho^{1}, \rho^{0}\right)$, is:

$$
\beta \mu^{1}\left(\rho^{0}, \rho^{1}\right)=\beta \mu_{i d}^{1}\left(\rho^{1}\right)+\beta \mu_{e x}^{1}\left(\rho^{0}, \rho^{1}\right),
$$

where the ideal gas contribution is taken as $\beta \mu_{i d}^{1}\left(\rho^{1}\right)=$ $\ln \left(\rho^{1} \sigma_{1}^{3}\right)$, the excess term $\beta \mu_{e x}^{1}\left(\rho^{0}, \rho^{1}\right)$ is taken in the form

$$
\begin{aligned}
\beta \mu_{e x}^{1}\left(\rho^{0}, \rho^{1}\right) & =\beta \mu_{e x}^{1}\left(\rho^{0}, \rho^{1}=0\right) \\
& -\int_{0}^{\rho_{1}} d \rho^{\prime} \int d \mathbf{R} c_{c}^{11}\left(R, \rho^{\prime}\right) .
\end{aligned}
$$

The second term in eq. (27) follows from the linear charging scheme applied in the functional relation between the chemical potential and adsorbed fluid density. The first term in the excess chemical potential, $\beta \mu_{i d}^{1}\left(\rho^{0}, \rho^{1}=0\right)$, is the excess chemical potential of a fluid component at infinite dilution in the mixture composed of matrix and fluid particles. The second term comes straightforwardly from the ROZ equation. It is an integral along density path (to a desired density) of the Fourier-transformed connected part of the fluid-fluid dcf (at $k=0$ ). The first term of the excess chemical potential, $\beta \mu_{e x}^{1}\left(\rho^{0}, \rho^{1}=0\right)$, is approximated using the theoretical Mansoori-CarnahanStarling-Leland equation of state for a mixture of hard spheres [54]. One can easily obtain the free energy of this mixture by integrating the equation of state and then by differentiation the chemical potential follows.

However, we also need to discuss how the attractive interactions between species can be treated. These interactions comprise an intrinsic feature of the realistic models for partially quenched fluid systems. In particular, the model for adsorption of methane in xerosilica gel of Kaminsky and Monson [55] is characterized by very strong attraction between matrix obstacles and fluid species. The fluid particles also interact so that attraction between them is present in the model. Moreover, both types of attraction (the fluid-matrix and fluidfluid) must be included to gain insight in the phase transitions in partly quenched media. The method of Ford and Glandt to obtain the chemical potential does not serve for this purpose unfortunately. It is well known that the hypernetted chain closure possesses a line of discontinuity of solutions while approaching the liquidvapor transition. However, this line cannot be attributed 
to the coexistence curve. On the other hand the PY closure also possesses its discontinuity line. Therefore one cannot perform integration by density in eq. (27) along path which can be of interest to study phase transitions.

The simplest tools to include the attractive interactions between species have been proposed by Ford and Glandt [23]. They have considered the mean field approximation (MFA) for the model in which fluid particles possess hard cores and, in addition, attract each other and with matrix species via finite ranged square-well potential [24], i.e.

$$
U^{11}(r)=\left\{\begin{array}{cc}
\infty, & r<\sigma_{1} \\
-\varepsilon_{f f}, & \sigma_{1}<r<\lambda_{f f} \sigma_{1} \\
0, & r>\lambda_{f f} \sigma_{1}
\end{array}\right.
$$

and

$$
U^{10}(r)=\left\{\begin{array}{cc}
\infty, & r<\sigma_{10} \\
-\varepsilon_{f m}, & \sigma_{1}<r<\lambda_{f m} \sigma_{10} \\
0, & r>\lambda_{f m} \sigma_{10}
\end{array}\right.
$$

where $\sigma_{10}=0.5\left(\sigma_{0}+\sigma_{1}\right)$ and $\lambda_{i j}$ describe the range of attractive wells with the depths $\varepsilon_{i j}$.

For this model, equations (26) and (27) have been used only to obtain the chemical potential of a reference system (a hard sphere fluid adsorbed in a hard sphere matrix). In the mean field approximation the contribution of square-well attractive forces into the chemical potential of fluid species is the following [24]

$$
\begin{aligned}
\beta \mu_{\text {attr }}^{1}\left(\rho^{1}, \rho^{0}\right)= & -\frac{4 \pi}{3} \rho^{1} \sigma_{1}^{3}\left(\lambda_{f f}^{3}-1\right) \varepsilon_{f f} \\
& -\frac{4 \pi}{3} \rho^{0} \sigma_{01}^{3}\left(\lambda_{f m}^{3}-1\right) \varepsilon_{f m}
\end{aligned}
$$

Resulting isotherms, $\beta \mu^{1}\left(\rho^{1}\right)$, permit to obtain completely the coexistence curve, if the Maxwell construction is used. However, this theoretical method of Ford and Glandt, i.e. the MFA for partly quenched systems, does not permit to gain insight into the influence of fluidfluid and fluid-matrix attraction separately on the coexistence of phases. Moreover, a simplified treatment of attractive forces does not permit to obtain a peculiar shape of the liquid-vapor coexistence line observed in computer experiment [28]. Kierlik et al. [26] have investigated the model with Lennard-Jones attractive forces between fluid particles and have shown that the mean spherical approximation (MSA) yields a qualitatively similar behavior to the MFA for adsorption isotherms, as expected. It has been shown, however, that the optimized random phase (ORPA) approximation (the MSA represents a particular case of this theory), if supplemented by the contribution of the second and third virial coefficients, yields the peculiar coexistence curve which is qualitatively similar to that observed in computer experiment [28]. Many unsolved issues remain, if one decides to apply the ORPA plus virial coefficients theory. Much criticism can appear. Nevertheless, this approach seems promising, at present, to develop thermodynamic description of partly quenched fluid systems with attractive forces.

We proceed with the description of the results which follow from some original applications of the ROZ methodology.

\section{APPLICATIONS OF THE ROZ THEORY FOR TWO-DIMENSIONAL AND THREE-DIMENSIONAL MODELS OF PARTLY QUENCHED SYSTEMS}

\section{A. Two-dimensional models for partly quenched systems}

First we would like to consider a simple model for quenched-annealed fluids and demonstrate the applications in practice of the theories presented above. Our focus in this section is the model for a partly quenched monomolecular adlayer, i.e. the adlayer which includes a rigid subsystem of matrix particles and fluid particles free to equilibrate in this environment. Our treatment concerns a two-dimensional (2D) model of this composite adlayer, because in many cases monomolecular adlayers are very similar to $2 \mathrm{D}$ fluids. On the other hand, the model is of interest in developing the theory of $2 \mathrm{D}$ phase transitions in partly quenched systems. Some of the results concerning $2 \mathrm{D}$ quenched-annealed models have been recently presented in Refs. [56, 57].

We consider a 2D model for a simple fluid adsorbed in a disordered quenched environment. The model consists of a hard disc fluid and a hard disc matrix. As in previous studies of partly quenched systems the species superscript ' 0 ' is for the matrix component and the species superscript ' 1 ' is for the fluid component. The matrix and fluid particles are considered at density $\rho^{0}$ and $\rho^{1}$, respectively. The diameters of the matrix and fluid particles are $\sigma_{0}$ and $\sigma_{1}$, respectively. Actually, the monolayer deposition of the matrix species can be considered at a given chemical potential. However, as the matrix is quenched, it is sufficient for our purposes to fix its density. The chemical potential of the adsorbed fluid will be obtained afterwards, using the solution of the 2D ROZ equations. The model for matrix and fluid species is defined as:

$$
U^{i j}(R)=\left\{\begin{array}{cc}
\infty, & R<\left(\sigma_{i}+\sigma_{j}\right) / 2 \\
0, & R>\left(\sigma_{i}+\sigma_{j}\right) / 2
\end{array},\right.
$$

where $i, j$, take values 0,1 . Obviously, all the distances above are two-dimensional. We assume that the matrix distribution can be prepared such to correspond to an equilibrium distribution of discs.

We obtain the pair correlation function for matrix species, $h^{00}(R)$, by solving eq. (21) for $2 \mathrm{D}$ case with Percus-Yevick closure

$$
c^{00}(R)=\left[\exp \left(-\beta U^{00}(R)\right)-1\right] y^{00}(R)
$$


where $y^{00}(R)$ is the cavity distribution function, $y^{00}(R)=\exp \left(\beta U^{00}(R)\right)\left[1+h^{00}(R)\right]$. Here and in equations below we employ the numerical method of solution, by using either direct iterations or in some cases the Labik-Malijevky-Vonka algorithm [58]. It is important to mention that in contrast to the three-dimensional cases, everywhere in $2 \mathrm{D}$ problems one must employ Hankel-Bessel transforms [59].

Next we solve eqs. (22) and (23) for 2D model in question. Two approximations have been used, namely the PY closure,

$$
\begin{gathered}
c^{10}(R)=\left[\exp \left(-\beta U^{10}(R)\right)-1\right] y^{10}(R), \\
c^{11}(R)=\left[\exp \left(-\beta U^{11}(R)\right)-1\right] y^{11}(R), \\
c^{11, b}(R)=0
\end{gathered}
$$

where $y^{10}(r)$ and $y^{11}(r)$ are the corresponding cavity distribution functions, and the hypernetted chain approximation

$$
\begin{gathered}
c^{10}(R)=\exp \left[-\beta U^{10}(R)+\gamma^{10}(r)\right]-1-\gamma^{10}(r), \\
c^{11}(R)=\exp \left[-\beta U^{11}(R)+\gamma^{11}(r)\right]-1-\gamma^{11}(r), \\
c^{11, b}(R)=\exp \left[\gamma^{11, b}(r)\right]-1-\gamma^{11, b}(r),
\end{gathered}
$$

where $\gamma^{i j}$ is the general notation for $h^{i j}-c^{i j}$. Having the solution of $2 \mathrm{D}$ ROZ equations available we proceed with the evaluation of the chemical potential of adsorbed fluid. We apply eqs. (26) and (27) to obtain the chemical potential. Note that the Hankel-transform is employed in eq. (27) for the system in question.

In order to obtain the chemical potential of adsorbed fluid at infinite dilution in the matrix of discs the following procedure has been performed. The starting point is the equation of state of Boublik for a mixture of hard discs [60]. It reads

$$
\beta P / \rho=1 /(1-\eta)+\gamma_{s} \eta /(1-\eta)^{2},
$$

where $\gamma_{s}=\left(\sum x_{i} \sigma_{i}\right)^{2} /\left(\sum x_{i} \sigma_{i}^{2}\right), x_{i}$ is the molar fraction of species $i, \rho$ and $\eta$ are the density and the packing fraction of the system made of a fluid component and matrix particles. Integrating this equation by density yields the free energy of the mixture. Then the chemical potential of a fluid component at infinite dilution in the matrix is obtained straightforwardly. It reads

$$
\begin{gathered}
\beta \mu_{e x}^{1}\left(\rho^{0}, \rho^{1}=0\right)=\eta^{0} /\left(1-\eta^{0}\right)-\ln \left(1-\eta^{0}\right)+ \\
\left(1-\eta^{0}\right)^{-2}\left(2-\eta^{0}\right) \eta^{0} \alpha_{1},
\end{gathered}
$$

where $\alpha_{1}=\sigma_{1}^{2} / \sigma_{0}^{2}$. We present some adsorption isotherms for the model under study in fig. 1. Higher matrix density lower adsorption, as expected. The effect of approximation becomes more pronounced at high val- ues for chemical potential, i.e. for high fluid densities. At low values of the chemical potential both approximations involved, the PY and HNC, yield almost indistinguishable results. The HNC approximation yields slightly lower adsorption for high values of the chemical potential. It is difficult to discuss for the moment the accuracy of each approximation quantitatively, in the absence of the Grand Canonical Monte Carlo computer simulation data for isotherms in question. However, the PY approximation seems more confidable, similarly to the case of three-dimensional systems [22]. What kind of helpful information one can extract from these adsorption isotherms? To answer this question we would like to note that the comparisons of the fluid structure in different matrices must be performed at given value of the chemical potential. Moreover, these adsorption isotherms can be used as a reference data for the development of the adsorption isotherms for systems which may include attractive and/or associative interactions between fluid particles as well as between them and matrix species.

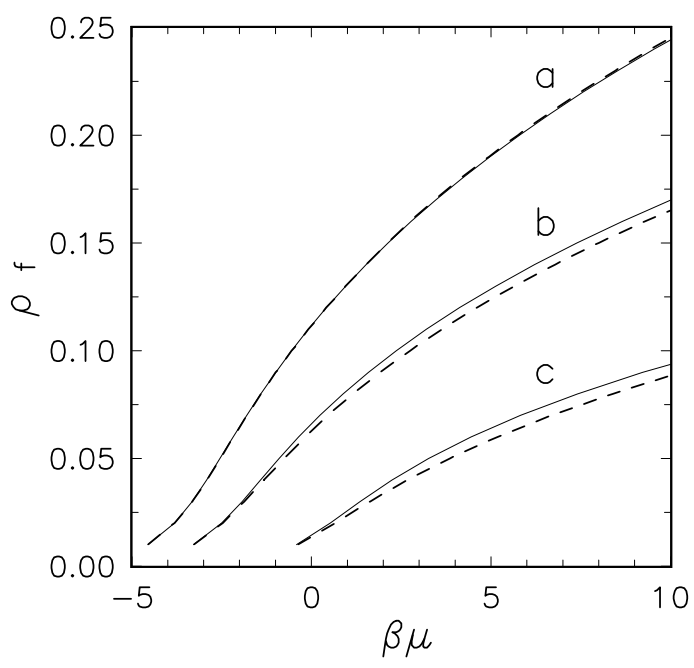

Fig. 1. Adsorption isotherms of a hard disc fluid in a disordered hard disc matrices of different density. The dashed and solid lines follow from the HNC and PY approximation for the connected direct correlation functions of fluid species. The curves from top to bottom are for $\rho^{0} \sigma_{0}^{2}=0.01, \rho^{0} \sigma_{0}^{2}=0.3$ and $\rho^{0} \sigma_{0}^{2}=0.6$. The matrix and fluid discs are of equal diameter.

Now we perform comparisons of the fluid structure in disordered matrix at intermediate density $\rho^{0}=0.3$ (fig. 2). The curves presented in that figure follow from the PY and HNC approximations. Besides, we have performed canonical simulations for the fluid density that is taken as an input to solve the ROZ equations. In the case of simulations thermal averages have been collected for at least 10 independent matrix configurations. By independent we mean configurations that are sufficiently distanced from each other during the productive part of the simulative run. We observe that adsorbed fluid is quite structured, as a result of the spatial confinement 
of pores. Especially the contact values of the distribution functions are higher that for the fluid at the same density in the bulk. The PY approximation is more successful in the description of the structure, in comparison with the HNC closure, especially at small interparticle separations. In fact, we are evaluating the accuracy of these closures to get an insight on the possibility to use them for more sophisticated systems than hard discs. It is of interest at the next step to include the attractive interactions into our model.
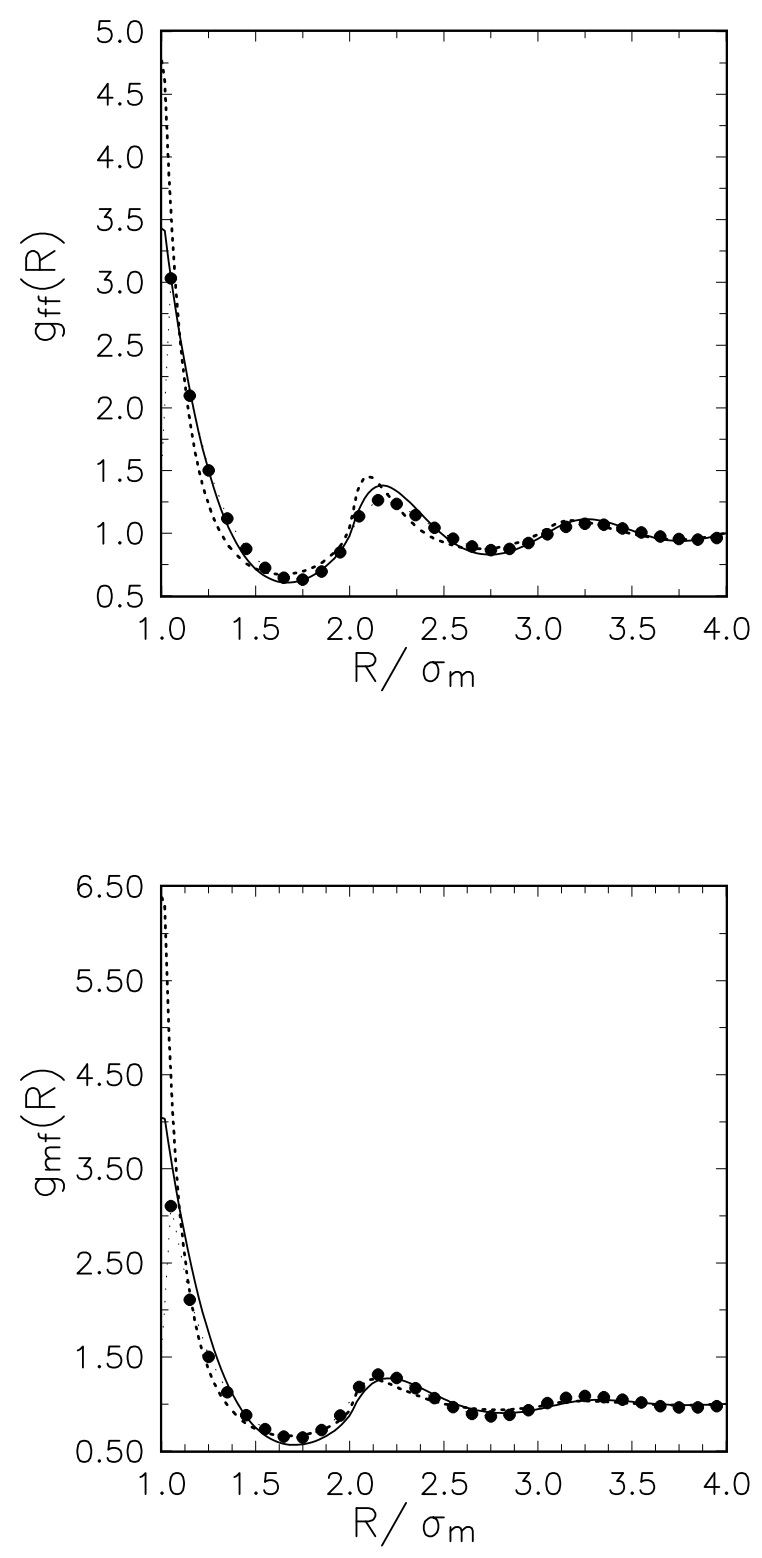

Fig. 2. A comparison of the fluid structure in terms of the fluid-fluid (part a) and fluid-matrix (part b) distribution functions of discs from the ROZ-HNC and ROZ-PY theory performed at $\rho^{0} \sigma_{0}^{2}=0.3$ and $\rho^{1} \sigma_{1}^{2}=0.4$. The matrix and fluid discs are of equal diameter. The nomenclature of the lines is like in fig. 1. Solid circles correspond to the results of Monte Carlo canonical simulations.

\section{B. On the effects of attractive interactions}

In this subsection we would like to discuss some results obtained for the model studied in the previous paragraph but with attractive interactions included. We assume that hard core fluid particles interact between themselves outside their core via square-well attractive forces, i.e the model for $U^{11}(R)$ is defined by eq. (28), but for $2 \mathrm{D}$ case. In a similar manner, the $U^{10}(R)$ interaction is chosen in the form given by eq. (29), but for $2 \mathrm{D}$ case. The chemical potential of the reference systems, i.e. of fluid discs adsorbed in a hard disc matrix, has been calculated using eqs. (26) and (27).

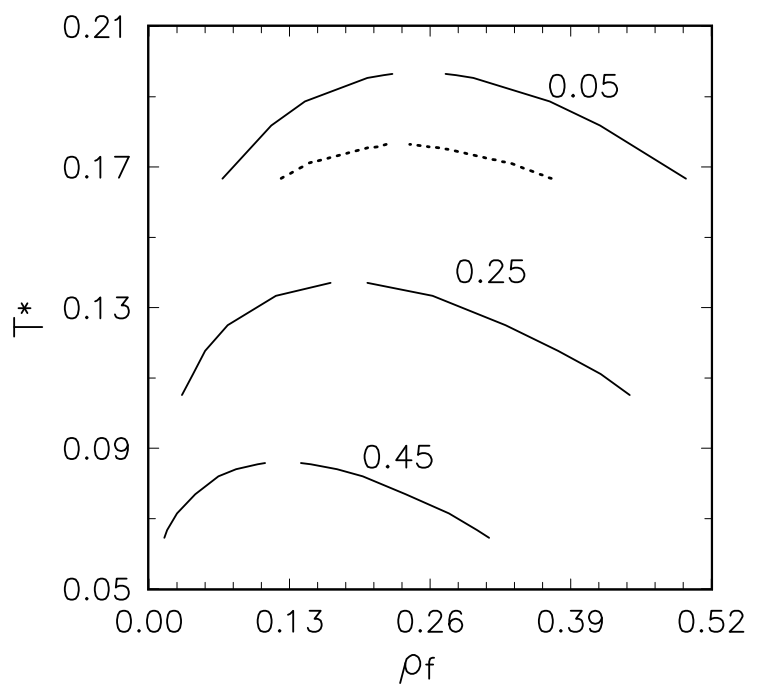

Fig. 3. Liquid-vapor coexistence curves for adsorbed fluid of discs with square-well attraction, like in fig. 3, in matrices at different density $\rho^{0} \sigma_{0}^{2}=0.05, \rho^{0} \sigma_{0}^{2}=0.25$ and $\rho^{0} \sigma_{0}^{2}=0.45$ from up to down. The width the attractive potential is $\lambda_{f f}=\lambda_{f m}=1.25$ and $T^{*}=1 / \beta \varepsilon_{a t t r}$. These results are for the model $\sigma_{0}=\sigma_{1}$. The dotted line shows the coexistence curve for the model $\sigma_{1}=0.9 \sigma_{0}$ with square well attraction like in the previous case, however, at matrix density $\rho^{0} \sigma_{0}^{2}=0.25$.

If the mean field approximation is used, the contribution from the attractive forces into the chemical potential reads

$$
\begin{aligned}
\beta \mu_{a t t r}^{1}\left(\rho^{1}, \rho^{0}\right)= & -\pi \rho^{1} \sigma_{1}^{2}\left(\lambda_{f f}^{2}-1\right) \varepsilon_{f f} \\
& -\pi \rho^{0} \sigma_{01}^{2}\left(\lambda_{f m}^{2}-1\right) \varepsilon_{f m} .
\end{aligned}
$$

For high temperatures the adsorption isotherms have been obtained monotonous. However, if the temperature lowers we observe that the adsorption isotherms exhibit the behavior characteristic for liquid-gas transition. Applying then the Maxwell construction for these isotherms, we obtain the coexistence curves for liquidvapor equilibrium in different matrices. The results are 
presented in fig. 3. We conclude that higher matrix density stronger suppresses the critical temperature. The critical density is less sensitive to the changes of matrix density. It decreases, however, with increasing matrix density. Actually, the coexistence curve shrinks with increasing matrix density. As expected, our results for the two-dimensional model are qualitatively similar to those obtained by Ford and Glandt [23] in the case of three-dimensional models with square-well attraction by using the mean field approximation. It is interesting to mention that the difference of diameters of fluid and matrix species may yield essential changes of the critical temperature of the system. This result may be of interest when studying the phase behavior of the monolayers of partly quenched systems. Nevertheless we plan in the future work to involve more sophisticated tools, than the mean filed approximation, to investigate twodimensional phase transitions.

\section{Three-dimensional models}

As an example of the application of ROZ equations for three-dimensional models we consider some of the results obtained in our recent study [61]. Namely, we have considered a model for a composite gel made of hard spheres of different diameters and have studied the adsorption of a hard sphere fluid in this matrix. The problem of adsorption of fluids in composite matrices is of importance for practical reasons, besides basic science. Evidently, the model is very simplified in comparison with reality. However, we expect the model to be of interest as a reference system to include a more sophisticated form of interparticle interactions.

We introduce, for the sake of convenience, species indices ' $a$ ' and ' $b$ ' for the components of the matrix component and species index 'f' for the fluid component. The matrix and fluid particles at density $\rho_{m}, \rho_{m}=\rho_{a}+\rho_{b}$, and $\rho_{f}$, respectively. The diameter of matrix and fluid particles is denoted by $\sigma_{a}, \sigma_{b}$ and $\sigma_{f}$, respectively. Actually the preparation of the matrix species can be considered at given chemical potentials for both matrix components. However, the matrix is quenched as a mixture (i.e. not by subsequent quenching of its each component) and it is sufficient for our purposes to fix the total matrix density and the concentration of one component, say $x_{a}=\rho_{a} / \rho_{m}$. The chemical potential of adsorbed fluid will be obtained afterwards, from the solution of the ROZ equations. Then we will be able to discuss the adsorption isotherms for annealed species.

The model for matrix and fluid species is defined as:

$$
U^{i j}(r)=\left\{\begin{array}{cc}
\infty, & r<\left(\sigma_{i}+\sigma_{j}\right) / 2 \\
0, & r>\left(\sigma_{i}+\sigma_{j}\right) / 2
\end{array},\right.
$$

where $i, j$, take values $a, b$, and $f$.

We assume that the matrix distribution can be prepared such to correspond to an equilibrium distribution of discs. Let us proceed to the ROZ equations. They read [16-18]:

$$
h^{m n}-c^{m n}=\sum_{i=a, b} c^{m i} \otimes \rho_{i} h^{i n}
$$

for the matrix subsystem (the superscripts $m, n$ take values $a$ and $b$ )

$$
\begin{aligned}
& h^{f a}-c^{f a}=\sum_{i=a, b} c^{f i} \otimes \rho_{i} h^{i a}+c^{f f(1)} \otimes \rho_{f} h^{f a}, \\
& h^{f b}-c^{f b}=\sum_{i=a, b} c^{f i} \otimes \rho_{i} h^{i b}+c^{f f(1)} \otimes \rho_{f} h^{f b},
\end{aligned}
$$

for the fluid-matrix correlations. Equations (23) for fluid-fluid correlations must be also modified to include both matrix components, it is straightforward to do that and for the sake of brevity we omit to write down these equations explicitly. We then solve the ROZ equations and calculate the adsorption isotherms according to eqs. (26), (27). Just to derive the first contribution into the excess chemical potential of a fluid at infinite dilution in a two-component matrix we apply the MansooriCarnahan-Starling-Leland equation of state for threecomponent mixture.

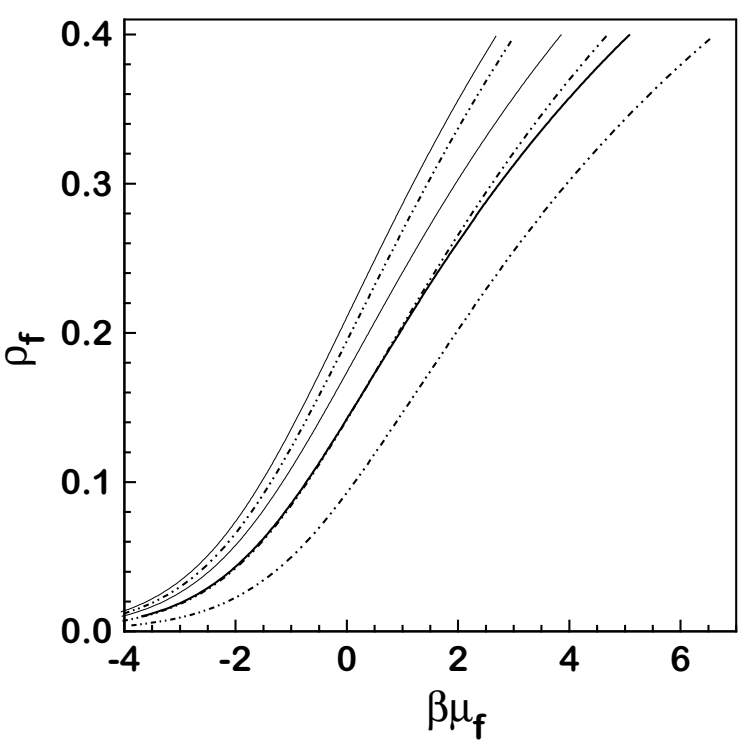

Fig. 4. Adsorption isotherms of a hard sphere fluid in a disordered hard sphere matrix with the packing fraction $\eta_{m}=0.13 ; 0.26$ and 0.39 (from left to the right). The matrix is made of the particles with the diameter $\sigma_{m}=7 \sigma_{f}$ (solid lines) and $\sigma_{m}=3 \sigma_{f}$ (dash-dotted lines).

We present adsorption isotherms for matrices at constant value of packing fraction but made of different particles with large diameter, $\sigma_{m}=7 \sigma_{f}$ (similarly to the 
model of Kaminsky and Monson) and of smaller diameter, $\sigma_{m}=3 \sigma_{f}$, in fig. 4 . As expected, the adsorption of a hard sphere fluid is slightly larger in a matrix made of large particles for all matrices in question. The difference in adsorption increases for denser matrices. Should the matrix be made of a mixture then the adsorption isotherms will be located in the intermediate region between two limiting cases. Evidently the effect of diameter of matrix particles is not substantial. However, in the presence of attractive interactions the matrix composition may have serious influence on the critical parameters of the system. Of particular importance may be the fluid-matrix attractive interactions. They can promote selective adsorption of fluid species w.r.t. each of matrix components and influence then the coexistence curve.

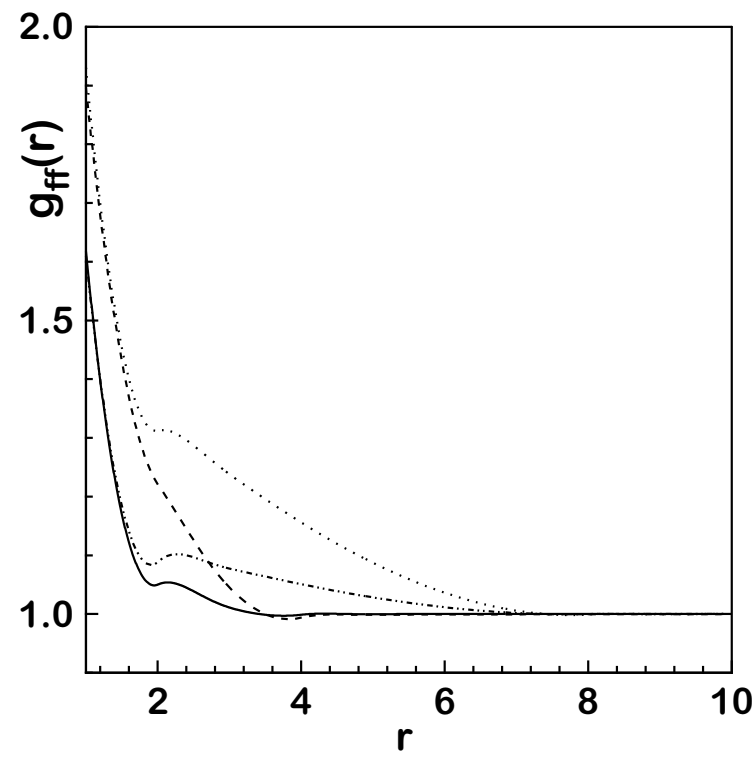

Fig. 5. A comparison of the fluid structure, at chemical potential $\beta \mu_{f}=0$, in terms of the fluid-fluid distribution functions in disordered matrices made of particles $\sigma_{m}=7 \sigma_{f}$ (solid lines) and $\sigma_{m}=3 \sigma_{f}$ (dash-dotted lines). The matrices are considered at packing fraction $\eta_{m}=0.13$ and 0.39 . Upper curves correspond to a denser matrix whereas lower curves correspond to a more dilute matrix.

Some examples of the structural properties, dependent on the matrix particles diameter are shown in fig. 5. We observe the following trends of behavior of the pair distribution functions. The contact value of the distribution function depends on the matrix density. The fluid is essentially 'compressed' in comparison to the bulk fluid at the same density. At larger distances we observe rapid degradation of correlations. The structures shown in this figure are very similar to those observed in the model of adsorption of methane in xerosilica gel. This similarity serves as the manifestation that excluded volume effects are of primary importance on the structure of adsorbed fluids.

\section{Adsorption of Lennard-Jones fluid in model xerosilica gel}

It is of interest to discuss more complicated models of adsorption of fluids in disordered porous media. In this subsection we turn our attention to Kaminsky and Monson model of silica xerogel [55]. The model is sufficiently realistic, however, remains computationally tractable using integral equations and simulations. In the case of adsorption of a Lennard-.Jones fluid in xerosilica gel the model is defined by the following interaction potentials:

$$
\begin{gathered}
U_{f f}(r)=4 \varepsilon\left[\left(\sigma_{f} / r\right)^{12}-\left(\sigma_{f} / r\right)^{6}\right], \\
U_{m m}(r)=\left\{\begin{array}{cc}
\infty, & r<7.055 \sigma_{f} \\
0, & r>7.055 \sigma_{f}
\end{array},\right. \\
U_{f m}(r)=\left\{\begin{array}{cc}
\infty, & r<0.5 \times 7.055 \sigma_{f} \\
\varphi(r), & r>0.5 \times 7.055 \sigma_{f}
\end{array},\right.
\end{gathered}
$$

where

$$
\begin{aligned}
\varphi(r) & =\frac{16}{3} \pi \rho_{s}\left(\frac{7.055 \sigma_{f}}{2}\right)^{3} \varepsilon_{g s}\left[\left(r^{6}+\frac{21}{5} r^{4} R^{2}\right.\right. \\
& \left.\left.+3 r^{2} R^{4}+\frac{R^{3}}{3}\right) \sigma_{g s}^{3}\left(r^{2}-R^{2}\right)^{-9}-\sigma_{g s}^{6}\left(r^{2}-R^{2}\right)^{-3}\right]
\end{aligned}
$$

with following set of parameters: $R=1.346 \mathrm{~nm}, \sigma_{g s}=$ $0.33 \mathrm{~nm}, \rho_{s}=44 \mathrm{~nm}^{-3}, \varepsilon_{g s} / k=339 \mathrm{~K}$. The model under consideration includes effects of confinement due to disordered porous media. Moreover it contains effects of wetting of the surface of matrix particles by fluid species. The model is quite difficult to treat using integral equations because of a high asymmetry of diameters of fluid and matrix species and due to strong attraction between them. However, the model has been successfully investigated in the framework of grand canonical Monte Carlo simulations [28]. Page and Monson have obtained two main conclusions.

The first of them concerns the behavior of LennardJones fluid in a matrix like above with fluid-matrix attraction switched off. It has been shown that two transitions between fluid phases in the systems are present. One of them can be associated with the vapor-liquid transition. As expected for the system under confinement, the coexistence region appears at lower temperatures comparing with the bulk, the critical density also is lower. In general, the coexistence curve is narrower than for the bulk fluid. The second coexistence region occurs at low temperature on the high density side of the vapor-liquid coexistence region. It is to some extent analogous to a predrying transition for a liquid in contact with a surface.

In the case when fluid-matrix attractive forces are present, i.e. in the model of Kaminsky and Monson, two transitions also are observed. The larger coexistence region can be associated with liquid-vapor transition. The coexistence curve is shifted to higher density than for the bulk. The second coexistence region is associated with 
the wetting behavior of adsorbed fluid. This transition occurs on the low-density side of the vapor-liquid coexistence curve.

These results of computer simulations have become a challenge for the theory of partly quenched systems. As yet the most successful attempt to obtain the coexistence curves like the described above has been undertaken by Kierlik et al. [26]. The model with which these authors have dealt with is simpler, in comparison with the one considered in the simulative study [27]. Namely the fluid of Lennard-.Jones particles, and with Lennard-.Jones interaction between fluid and matrix particles, have been considered to be adsorbed in an equilibrium configuration of hard spheres. The simplifying assumption was that diameters of both species are equal. The model of randomly centered spheres, considered as a matrix, also yields the coexistence curve of a specific shape [27].

The optimized random phase approximation (ORPA) has been used to describe an equilibrium mixture of $s$ replicas of the adsorbed fluid subsystem and matrix species. Then a limit $s \rightarrow 0$ has been performed explicitly to yield the free energy of partly quenched system. We do not present it explicitly referring to the original paper [27]. Most importantly the ORPA term for the free energy has been supplemented by the contribution of the second and third virial coefficient. In fact, these additional contributions provide the appearance of two phase transitions that seem qualitatively similar to those observed in the simulative study. A disappointing feature of the results obtained is that the coexistence curve is very sensitive to the approximations applied, the convergence of the optimized cluster series is quite poor in the region of densities of interest. These problem may become even more severe for the model of adsorption of Lennard-Jones fluids in model xerosilica gel. Probably a resummation of some terms in different virial coefficients would be helpful. Our preliminary investigations of the phase behavior of a one-component Yukawa fluid show that this may be promising [62]. Nevertheless, intensive studies in this direction are required.

\section{THEORY OF PARTLY QUENCHED INHOMOGENEOUS FLUIDS}

To our best knowledge there was only one attempt to consider inhomogeneous fluids adsorbed in disordered porous media [30] before our recent studies [31, 32]. Inhomogeneous Replica Ornstein-Zernike equations, complemented by either Born-Green-Yvon (BGY), or LovettMou-Buff-Wertheim (LMBW) equation for density profiles, have been proposed to study adsorption of a fluid near a plane boundary of a disordered matrix, which has been assumed uniform in a half-space [30]. However, the theory has not been complemented by any numerical solution. Our main goal is to consider a simple model for adsorption of a simple fluid in confined porous media proposed by us recently and to solve it. In this section we follow our works $[31,32]$.

First we are looking for the adsorption of a fluid consisting of particles of species $m$, in a slit-like pore of the width $H$. The pore walls are chosen normal to the $z$ axis and the pore is centered at $z=0$. Adsorption of the fluid $m$, i.e. the matrix, occurs at equilibrium with its bulk counterpart at the chemical potential $\mu_{m}$. The matrix fluid is then characterized by the density profile $\rho_{m}(z)$ and by the inhomogeneous pair correlation function $h_{m m}(1,2)$. The structure of that fluid is considered quenched at a state determined by $\mu_{m}$, thus a confined porous medium is formed.

Now, we would like to investigate adsorption of another fluid of species $f$ in the pore filled by the matrix. The fluid $f$, outside the pore has the chemical potential $\mu_{f}$; at equilibrium the adsorbed fluid $f$ reaches the density distribution $\rho_{f}(z)$. The pair distribution of $f$ particles is characterized by the inhomogeneous correlation function $h_{f f}(1,2)$. The matrix and fluid species are denoted by 0 and 1 . We assume the simplest form of the interactions between particles and between particles and pore walls, choosing both species as hard spheres of unit diameter,

$$
\begin{aligned}
& U_{i j}(r)=\left\{\begin{array}{cc}
\infty, & r<1 \\
0, & r>1
\end{array} ;\right. \\
& U(z)=\left\{\begin{array}{cc}
\infty, & z<0.5|H-1| \\
0, & \text { otherwise }
\end{array} .\right.
\end{aligned}
$$

where $i, j$ are species indices. The evaluation of the matrix structure is irrelevant to the procedure below; it is obtained using the second order Ornstein-Zernike (OZ2) equation

$$
h_{00}(1,2)-c_{00}(1,2)=\int d 3 \rho_{0}\left(z_{3}\right) c_{00}(1,3) h_{00}(3,2)
$$

supplemented by the LMBW equation for the density profile (DP)

$$
\frac{\partial \ln \rho_{1}\left(z_{1}\right)}{\partial z_{1}}+\frac{\partial \beta U\left(z_{1}\right)}{\partial z_{1}}=\int d 2 c_{00}(1,2) \frac{\partial \rho_{0}\left(z_{2}\right)}{\partial z_{2}}
$$

and the second order Percus-Yevick (PY2) closure

$$
y_{00}(1,2)=1+h_{00}(1,2)-c_{00}(1,2) \text {. }
$$

where $y_{00}(1,2)$ is the inhomogeneous cavity distribution function. The solution of the problem comprising eqs. (45) $-(47)$ yields $\rho_{0}(z)$ and $h_{00}(1,2)$, such that the one-particle cavity distribution function $y_{0}(z), y_{0}(z)=$ $\rho_{0}(z) \exp [\beta U(z)]$, outside the pore tends to its limiting value, determined by the configurational chemical potential, $y_{0}(z \rightarrow \pm \infty)=\exp \left(\beta \mu_{0}\right)$. The IROZ equations represent the essence of the procedure. They are $[30,31]$ 


$$
\begin{aligned}
& h_{10}(1,2)-c_{10}(1,2)=\int d 3 \rho_{0}\left(z_{3}\right) c_{10}(1,3) h_{00}(3,2)+\int d 3 \rho_{1}\left(z_{3}\right) c_{c, 11}(1,3) h_{10}(3,2) \\
& h_{01}(1,2)-c_{01}(1,2)=\int d 3 \rho_{0}\left(z_{3}\right) c_{00}(1,3) h_{01}(3,2)+\int d 3 \rho_{1}\left(z_{3}\right) c_{01}(1,3) h_{c, 11}(3,2) \\
& h_{11}(1,2)-c_{11}(1,2)=\int d 3 \rho_{0}\left(z_{3}\right) c_{10}(1,3) h_{01}(3,2)+\int d 3 \rho_{1}\left(z_{3}\right) c_{c, 11}(1,3) h_{11}(3,2)+\int d 3 \rho_{1}\left(z_{3}\right) c_{b, 11}(1,3) h_{c, 11}(3,2) \\
& h_{c, 11}(1,2)-c_{c, 11}(1,2)=\int d 3 \rho_{1}\left(z_{3}\right) c_{c, 11}(1,3) h_{c, 11}(3,2)
\end{aligned}
$$

The fluid-fluid pair $(h)$ and direct $(c)$ correlation functions consist of the blocking and connected part, $\varphi_{11}(1,2)=$ $\varphi_{b, 11}(1,2)+\varphi_{c, 11}(1,2)$, where $\varphi$ stands for $h$ and $c$, as appropriate. Similarly to [30], the BGY equation is used to relate the DPs with the pair functions,

$$
\frac{\partial \ln \rho_{1}\left(z_{1}\right)}{\partial z_{1}}+\beta \frac{\partial w\left(z_{1}\right)}{\partial z_{1}}=-\beta \int d 2 \rho_{1}\left(z_{2}\right) g_{11}(1,2) \frac{\partial U_{11}(12)}{\partial z_{2}}
$$

where $g_{11}(1,2)=1+h_{11}(1,2)$, and the effective onebody potential satisfies the relation,

$$
\frac{\partial w\left(z_{1}\right)}{\partial z_{1}}=\frac{\partial U\left(z_{1}\right)}{\partial z_{1}}+\int d 2 \rho_{0}\left(z_{2}\right) g_{10}(1,2) \frac{\partial U_{10}(12)}{\partial z_{2}}
$$

and where $g_{10}(1,2)=1+h_{10}(1,2)$.

Finally, the closure relations for the inhomogeneous pair functions must be chosen. The analysis of Stell and Given [16-18] has shown that the hypernetted chain closure is consistent with the ROZ equations for fluids in homogeneous disordered matrices while the PY closure belongs to a class of approximations used previously by Madden and Glandt [14] and is not consistent in this respect. The PY approximation for the fluid-fluid direct correlation function presumes that its blocking part vanishes. It implies that $c_{b, 11}(i, j)=0$, and

$$
y_{i j}(1,2)=1+h_{i j}(1,2)-c_{i j}(1,2)
$$

for $(i, j)=(1,0)$ and $(1,1)$. On the other hand, the inhomogeneous, or second order, hypernetted chain (HNC2) approximation reads

$$
\begin{aligned}
c_{b, 11}(i, j) & =\exp \left\{h_{b, 11}(i, j)-c_{b, 11}(i, j)\right\}-1 \\
& -\left\{h_{b, 11}(i, j)-c_{b, 11}(i, j)\right\}
\end{aligned}
$$

for the blocking term of the fluid-fluid function, and

$$
y_{i j}(1,2)=\exp \left\{h_{i j}(1,2)-c_{i j}(1,2)\right\}
$$

for $(i, j)=(1,0)$ and $(1,1)$.

In the numerical solution the matrix structure is evaluated form eqs. (45)-(47). Then eqs. (48)-(50) with corresponding closure approximations are solved. Details of the solution have been presented in [31, 32]. Briefly, the numerical algorithm uses an expansion of the two-particle functions into a Fourier-Bessel series. The three-fold integrations are reduced then to sums of one-dimensional integrations. In the case of hardsphere potentials, the BGY equation contains the deltafunction due to the derivative of the pair interactions. Therefore the integral in eqs. (49) and (50) are onefold and contain the "contact" values of the functions $g_{i j}\left(z_{1}, z_{2}, \sqrt{R^{2}+z_{12}^{2}}=1\right)$ for $(i, j)=(1,0)$ and $(1,1)$; these values have been evaluated by interpolation. As is often the case, the convergence of the numerical scheme is more difficult for the $\mathrm{HNC} 2$ closure than for the PY2 closure.

In order to test the theory, we have performed grand canonical ensemble Monte Carlo (GCMC) simulations. A rectangular simulative cell of dimensions $X L \times Y L \times H$ with periodic boundary conditions in the plane parallel to the pore walls has been used. The simulations consist of two steps. First, the grand canonical ensemble technique is used to fill the pore with the hard sphere matrix. After equilibration, a configuration of matrix particles whose number of particles corresponds to the average number of particles at the given chemical potential is selected and the second step of the simulations is started. During this step, we perform grand canonical ensemble simulations of the fluid in a pore filled with the matrix species.

The simulations are repeated several times starting from different matrix configurations. We have found that about 10 replicas of the matrix usually assures good statistics for the determination of the local fluid density. However, the evaluation of the nonuniform pair distribution functions requires much longer runs; at least 100 matrix replicas are needed to calculate the pair correlation functions for particles parallel to the pore walls. 
However, even as many as 500 replicas do not ensure the convergence of the simulative results for perpendicular configurations.
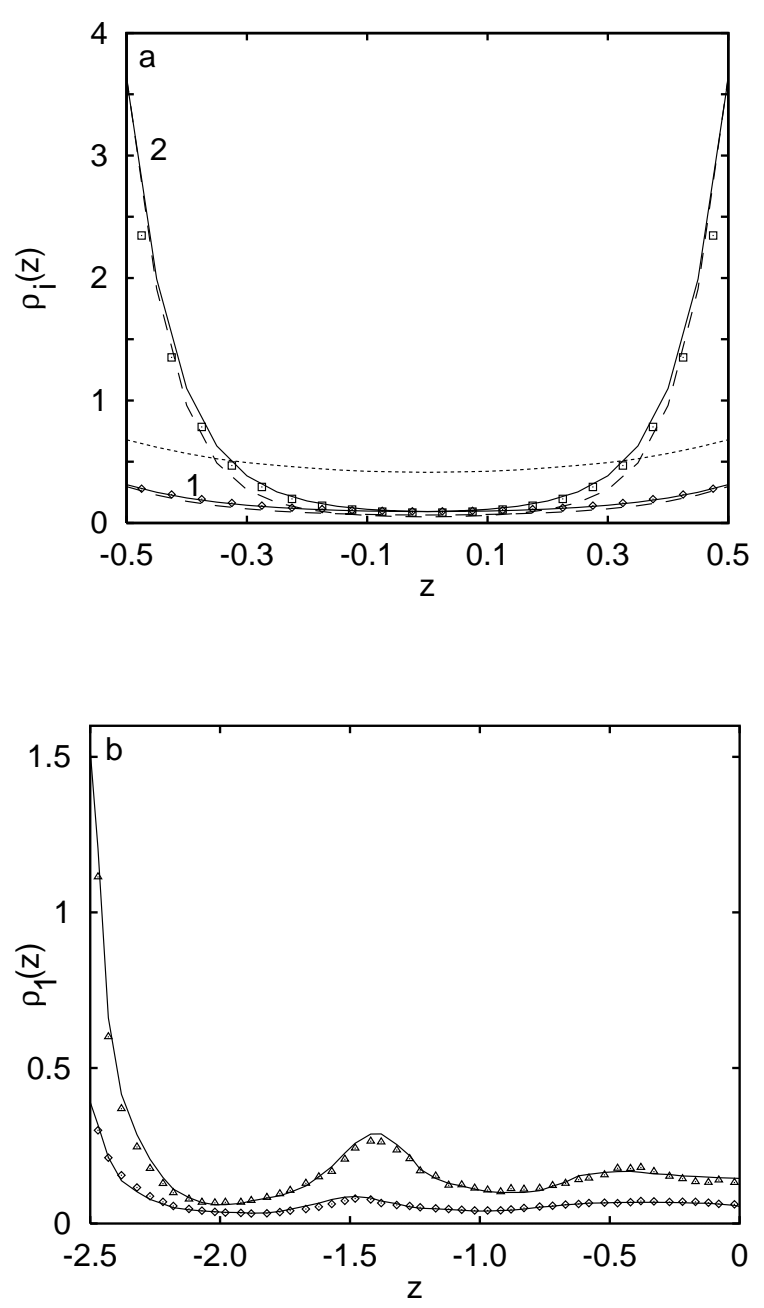

Fig. 6. Part a. A comparison of the simulated (GCMC) and theoretical (ROZ2+BGY+HNC2 and PY2 approximations) density profiles, $\rho_{1}(z)$, of an adsorbed fluid and in a disordered inhomogeneous matrix in a slit-like pore of the width $\mathrm{H}=2$. The chemical potential of the matrix particles is $\beta \mu_{0}=0.935$. The curves labeled 1 and 2 are for the chemical potential of fluid species $\beta \mu_{1}=0.935$, and 5.8346, respectively. The $\mathrm{PY} 2, \mathrm{HNC} 2$, and Monte Carlo results are given by the solid and dashed lines, and the symbols, respectively. The dotted line corresponds to the density profile of fixed obstacles, $\rho_{0}(z)$. Part b. The HNC2 and GCMC results for the density profiles for the case of a wide pore, $\mathrm{H}=6$. The chemical potential of the matrix species is $\beta \mu_{0}=4.8147$; the chemical potential of the fluid species is $\beta \mu_{1}=3.1136$ (lower curves and symbols) and $\beta \mu_{1}=7.0026$ (upper curve and symbols).

We present some of the results obtained for equal sized hard-sphere fluid and hard-sphere matrix in figs. 6 and 7. In these figures we show the density profiles and pair distribution functions of a fluid adsorbed in a pore filled with quenched matrix. Figure 6 shows the density profiles obtained for $\beta \mu_{0}=0.935$ (corresponding to the bulk fluid density $\rho_{0} \sigma_{0}^{3}=0.25$ when Carnahan-Starling equation of state is used) and $H=2 \sigma_{0}$ (part a) and for $\beta \mu_{0}=4.8147$ (corresponding to the bulk density equal to $\left.\rho_{0} \sigma_{0}^{3}=0.5\right)$ and for $H=6 \sigma_{0}$ (part b).

Let us first analyze the results for $H=2 \sigma$. At $\beta \mu_{0}=$ 0.935 the matrix density distribution, $\rho_{0}(z)$, in the entire pore is almost uniform. Consequently, the distribution of empty space throughout the pore is almost homogeneous. When the chemical potential of fluid species, $\mu_{1}$, increases, the density of fluid close to the pore walls increases substantially, whereas in the pore center $\rho_{1}(z)$ remains almost independent of $\mu_{1}$. Both theories, HNC2 and PY2, yield similar results for the density profiles and both agree well with the GCMC data. However, some discrepancies between the theory and simulations are observed close to the walls in a narrow pore. The absence of a large difference between the $\mathrm{PY} 2$ and $\mathrm{HNC} 2$ theories indicates that blocking effects due to the presence of the matrix are not essential.

We have also studied fluid distribution in the pore $H=6 \sigma_{0}$ (fig. $6 \mathrm{~b}$ ) at $\beta \mu_{0}=4.8147$ and at two values of $\beta \mu_{1}$, namely at $3.1136\left(\rho_{1} \sigma_{1}^{3}=0.4\right)$ and at 7.0026 $\left(\rho_{0} \sigma_{0}^{3}=0.7\right.$; fig. $\left.6 \mathrm{~b}\right)$. In this pore, we observe layering of the adsorbed fluid at high values of the chemical potential, $\beta \mu_{1}$. The maxima of the density profile $\rho_{1}(z)$ occur at distances that correspond to the diameter of fluid particles. With an increase of the fluid chemical potential, the pore filling takes place primarily at pore walls, but second-order maxima on the density profile $\rho_{1}(z)$ are also observed. The theory reproduces the computer simulation results quite well.

Let us show now the results obtained for inhomogeneous pair correlation functions. In figs. $7 \mathrm{a}$ and $7 \mathrm{~b}$ we present the projections of the pair correlation functions for particles parallel to the pore walls in the plane of the closest approach to the walls, $g_{i j}^{\|, w}$, and in the pore center, $g_{i j}^{\|, c}$ for the pore width $H=3 \sigma_{0}$. We observe that parallel correlations between the fluid particles are stronger in the pore center than at the pore walls. In contrast, the fluid-matrix parallel correlations are slightly stronger in the plane of the closest approach, due to the higher value of the matrix density at the walls. The theoretical approximations ( $\mathrm{HNC} 2$ and $\mathrm{PY} 2$ ) agree well with the simulation data. The $\mathrm{HNC} 2$ approximation yields slightly higher contact values for the pair correlation functions at the contact than does the PY2 approximation.

The presence of pore-fluid attractive forces changes the distribution of the particles inside the pore. The second series of our calculations has been performed for the case when hard spheres of matrix and fluid species interact with the pore wall by a hard sphere potential with an attractive tail

$$
U_{i}(z)=\left\{\begin{array}{cc}
\infty, & z<0.5\left|H-\sigma_{i}\right| \\
-\varepsilon\left[\frac{1}{z^{3}}+\frac{1}{(H-z)^{3}}\right], & \text { otherwise. }
\end{array}\right.
$$


Obviously, for $\varepsilon=0$ this interaction reduces to the one we have used above in this section. In our calculations we have chosen $\beta \varepsilon=3$ and, similarly to previous developments, the diameters of matrix and fluid species were taken to be equal, $\sigma_{0}=\sigma_{1}=1$.
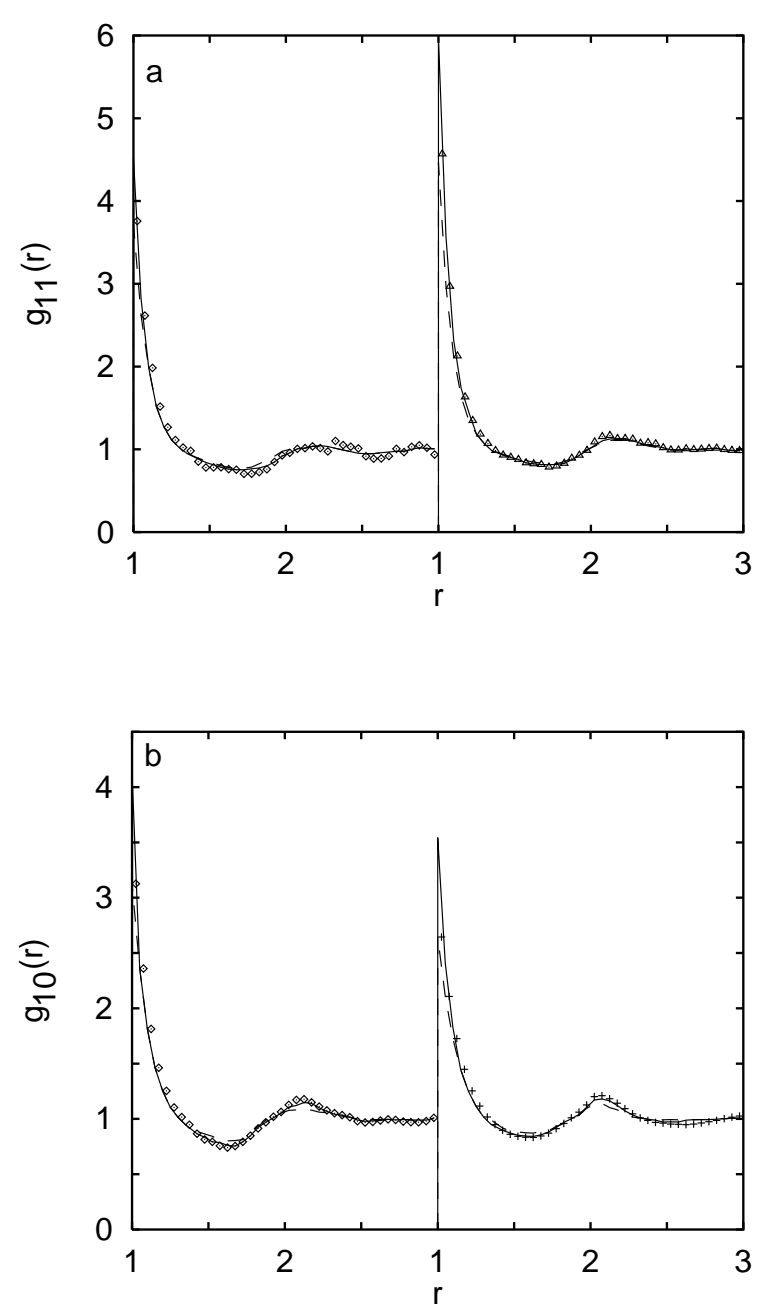

Fig. 7. Part a. A comparison of the fluid-fluid inhomogeneous pair distribution functions, $g_{11}(1,2)$ obtained using the $\mathrm{HNC} 2$ and PY2 approximations in the ROZ2+BGY equations, with GCMC results. The functions $g_{11}^{l l, w}$ (left panel) and $g_{11}^{\|, c}$ (right panel) are for the fluid particles in a parallel configuration in the plane of closest approach to the pore walls and in the pore center. The $\mathrm{HNC} 2$ and PY2 results are given by the solid and dashed lines, respectively. The GCMC results are given as symbols. The fluid and matrix particles are of equal size. The chemical potentials of matrix and fluid species are $\beta \mu_{0}=3.1136$ and $\beta \mu_{1}=8.3530\left(\rho_{0} \sigma_{0}^{3}=0.75\right)$. The pore width is $H=3 \sigma_{0}$. Part $\mathrm{b}$. The same as in part a, but for the fluid-matrix inhomogeneous pair distribution function.

Density profiles for pore widths $H=3 \sigma_{0}$ and $H=4 \sigma_{0}$ are given in figs. $8 \mathrm{a}$ and $8 \mathrm{~b}$, respectively. The fluid profiles have been evaluated from the solution of the $\mathrm{HNC} 2$ equation for $\beta \mu_{0}=0.935$ and $\beta \mu_{1}=3.1136$ (the system I) and for $\beta \mu_{0}=3.1136$ and $\beta \mu_{1}=8.3530$ (the system II). For comparison, the simulation values are also shown. The average matrix density in the pore, computed from the GCMC simulation values are 0.37 and 0.52 for the systems I and II, respectively. The corresponding values for average fluid density in the pores are 0.18 and 0.13 . Thus, high matrix density lowers fluid adsorption. The results shown in fig. $8 \mathrm{~b}$ correspond to those in figs. $8 \mathrm{a}$, except that $H=4$. The average matrix densities in the pore are 0.36 and 0.51 , respectively, for the systems I and II, whereas for the same systems the average fluid densities are 0.19 and 0.15 , respectively. The agreement of the calculated and simulated density profiles is satisfactory but not as good as was for the case of purely repulsive interaction $\beta \varepsilon=0$, cf. fig. 6 .
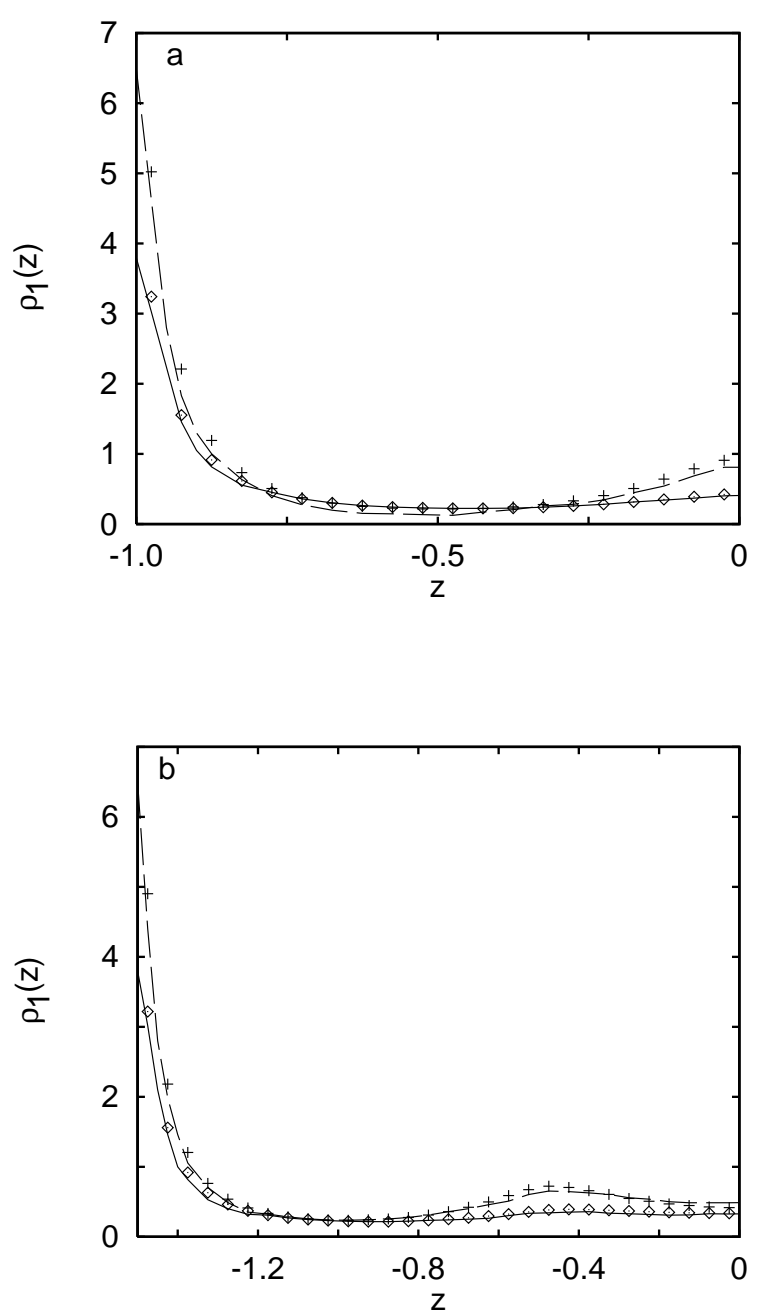

Fig. 8. Density profiles fluid spheres in a slit-pore for which $H=3 \sigma_{0}$ (part a) and $H=4 \sigma_{0}$ (part b) and with an attractive interaction between the spheres and the pore wall. The curves give the $\mathrm{HNC} 2$ results and the points give the simulation results, respectively. The lower curves are for system I, whereas the upper curves are for system II (for the system abbreviation see the text). 
The theory presented in this section is based on the grand canonical ensemble description, that is perfectly well-suited for the description of confined systems. Undoubtedly, in the case of attractive-repulsive interparticle forces this kind of approach may yield unexpected and rich structural and thermodynamic behavior in partly quenched confined systems.

Now we proceed with the application of the ROZ methodology for complex fluids. In general complex fluids adsorption in disordered media has been studied rarely [34]. Our intention is to develop the ROZ approach for chemically associating fluids.

\section{THE ASSOCIATIVE EXTENSION OF THE ROZ METHODOLOGY FOR PARTLY QUENCHED CHEMICALLY ASSOCIATING FLUIDS}

\section{A. A model for chemically associating fluid in disordered porous media}

In this part of the work our main concern is to review recent theoretical developments for partly quenched chemically associating fluids. We would like to consider only few simple models for chemically associating fluid adsorbed in a disordered quenched environment. The applications which involve the ideas of chemical association to generate matrix structure also have been studied [44].

The simplest model for associating fluids adsorption is designed to describe a dimerizing fluid in a hard sphere matrix. Similarly to the presentation above species superscript ' 0 ' is for the matrix component and superscript ' 1 ' is for the fluid component. We reserve subscripts to classify the bonding states in the theory of Wertheim for chemical association $[37,38]$. The matrix and fluid particle densities are denoted as $\rho^{0}$ and $\rho^{1}$, respectively. The diameter of matrix and fluid particles is by $\sigma_{0}$ and $\sigma_{1}$, respectively.

The model for a dimerizing fluid is defined by the following potentials [63]

$$
U^{11}(12)=U_{\text {non }}^{11}(r)+U_{\text {as }}^{11}\left(x_{12}\right) \text {, }
$$

where the first and second terms denote the nonassociative and associative contribution into the fluid-fluid interaction, respectively. The nonassociative term is chosen in the form

$$
U_{n \circ n}^{11}(r)=\left\{\begin{array}{cc}
\infty, & r<\sigma_{1} \\
0, & r>\sigma_{1}
\end{array}\right.
$$

The associative term in the fluid-fluid interaction has the form [63]

$$
U_{a s}^{11}(x)=\left\{\begin{array}{cc}
-\varepsilon_{a s}, & x<a \\
0, & x>a
\end{array},\right.
$$

where $\varepsilon_{a s}$ is the associative energy, a denotes the range of associative interaction; $x_{12}=\left|\mathbf{r}_{12}+\mathbf{d}\left(\vartheta_{1}\right)-\mathbf{d}\left(\vartheta_{2}\right)\right|$, where $\mathbf{d}\left(\vartheta_{1}\right)$ denotes the position and orientation of the attractive site on the surface of the repulsive core of molecule 1.

The geometric parameters of associative interaction $d$, $a$ for a dimerizing model, must satisfy saturation condition, namely, $\sigma_{1}<2 d+a<\sigma_{1}+(2-\sqrt{3}) d$, [63]. In our theoretical treatment we have chosen slightly smaller bonding length such that outer shell of the association site coincides with the surface of a hard sphere, then two spheres can form dimers while touching and also with negligible degree of overlap.

The matrix-matrix and fluid matrix interactions are chosen using the model of additive hard spheres, i.e.

$$
U^{00}(r)=\left\{\begin{array}{cc}
\infty, & r<\sigma_{0} \\
0, & r>\sigma_{0}
\end{array}\right.
$$

and

$$
U^{10}(r)=\left\{\begin{array}{cc}
\infty, & r<\left(\sigma_{0}+\sigma_{1}\right) / 2 \\
0, & r>\left(\sigma_{0}+\sigma_{1}\right) / 2
\end{array},\right.
$$

respectively. Let us define now the Mayer functions

$$
\begin{aligned}
f^{00}(r) & =\exp \left[-\beta U^{00}(r)\right]-1, \\
f^{10}(r) & =\exp \left[-\beta U^{10}(r)\right]-1, \\
f_{n o n}^{11}(r) & =\exp \left[-\beta U_{n o n}^{11}(r)\right]-1,
\end{aligned}
$$

and the associative 'Mayer' function [37],

$$
F_{a s}^{11}(12)=\exp \left[-\beta U_{\text {non }}^{11}\left(r_{12}\right)\right]\left\{\exp \left[-\beta U_{a s}^{11}\left(x_{12}\right)\right]-1\right\}
$$

In the theory of Wertheim only its orientation-averaged form is needed

$$
F_{a s}^{11}\left(r_{12}\right)=\int d \vartheta_{1} d \vartheta_{2} F_{a s}^{11}(12)=\left\{\begin{array}{cc}
0, & r_{12}<\sigma_{1} \\
0, & r_{12}>2 d+a \\
\frac{1}{24} \exp \left(\beta \varepsilon_{a s}\right)\left(a+2 d-r_{12}\right)^{2}\left(a-d+0.5 r_{12}\right) / d^{2} r_{12}, & \sigma_{1}<r_{12}<2 d+a
\end{array}\right.
$$


These ingredients are necessary for the application of the associative ROZ equations.

\section{B. Associative Replica Ornstein-Zernike equations and closure relations}

Let us assume that the matrix distribution corresponds to an equilibrium distribution of nonassociating hard spheres. Extending then the ROZ equations for nonassociating system to the case of associating fluid we obtain the associative ROZ equations. They read:

$$
h^{00}-c^{00}=c^{00} \otimes \rho^{0} h^{00}
$$

for the matrix subsystem,

$$
h_{\alpha}^{10}-c_{\alpha}^{10}=c_{\alpha}^{10} \otimes \rho^{0} h^{00}+\sum_{\mu \nu} c_{\alpha \mu}^{11(1)} \otimes \rho_{\mu \nu}^{1} h_{\nu}^{10}
$$

for the fluid-matrix correlations, and

$$
\begin{aligned}
& h_{\alpha \beta}^{11}-c_{\alpha \beta}^{11}=c_{\alpha}^{10} \otimes \rho^{0} h_{\beta}^{10}+\sum_{\mu \nu} c_{\alpha \mu}^{11(1)} \otimes \rho_{\mu \nu}^{1} h_{\nu \beta}^{11} \\
&+\sum_{\mu \nu} c_{\alpha \mu}^{11(2)} \otimes \rho_{\mu \nu}^{1} h_{\nu \beta}^{11(1)} \\
& h_{\alpha \beta}^{11(1)}-c_{\alpha \beta}^{11(1)}=\sum_{\mu \nu} c_{\alpha \mu}^{11(1)} \otimes \rho_{\mu \nu}^{1} h_{\nu}^{11(1)},
\end{aligned}
$$

for fluid-fluid correlations. Here $\otimes$ denotes convolution and $r$-dependencies are omitted for brevity. In eqs. (64)(66) we have used standard decomposition of the pair and direct fluid-fluid correlation functions into connected, $\phi_{\alpha \beta}^{11(1)}$, and blocking, $\phi_{\alpha \beta}^{11(2)}$, parts ( $\phi$ stands for $h$ and $c$ as appropriate) such that each function consists of two terms: $\phi_{\alpha \beta}^{11}=\phi_{\alpha \beta}^{11(1)}+\phi_{\alpha \beta}^{11(2)}$. The correlation functions which figure out in eqs. (64)-(66) are the partial correlation functions similar to those in the theory of Wertheim for chemical association [37, 38, 63].

In the case of dimerization, which we consider in the present work, lower indices in the correlation functions take the values ' 0 ' and ' 1 ', dependent on the bonding state of a fluid particle (it can be either free or partici- pate in a dimer). The symmetry relations for the correlation functions imply $\phi_{10}^{11}=\phi_{01}^{11} ; \phi_{\alpha}^{10}=\phi_{\alpha}^{01}$. The matrix of fluid density in the case of dimerization has the following form:

$$
\rho^{1}=\left(\begin{array}{cc}
\rho^{1} & \rho_{0}^{1} \\
\rho_{0}^{1} & 0
\end{array}\right)
$$

where $\rho_{0}^{1}$ is the density of unbounded fluid particles.

The partial pair correlation functions, $h_{\alpha \beta}^{11}$, yield the usual total pair correlation function (pcf) for fluid species via the following relation [63]

$$
h^{11}(r)=h_{00}^{11}(r)+2\left(\rho_{0}^{1} / \rho^{1}\right) h_{10}^{11}(r)+\left(\rho_{0}^{1} / \rho^{1}\right)^{2} h_{11}^{11}(r) .
$$

In the similar manner, the total fluid-matrix correlation function is defined as

$$
h^{10}(r)=h_{0}^{10}(r)+\left(\rho_{0}^{1} / \rho^{1}\right) h_{1}^{10}(r) .
$$

Worth mentioning that for the direct correlation function (dcf) we do not have similar relation, the partial dcfs are just defined but not determined via Wertheim's OZ equation; in our special case they are defined by the AROZ equations. The AROZ equation must be supplemented by the self-consistency relation for fluid density. It reads $[37,63]$

$$
\rho^{1}=\rho_{0}^{1}+\left(\rho_{0}^{1}\right)^{2} \int d \mathbf{r} F_{a s}(r) y_{00}^{11}(r),
$$

where $y_{00}^{11}(R)$ is the partial cavity distribution function for unbounded fluid species.

Let us now consider the closure relations for AROZ equations. For the sake of convenience, let us introduce the notation $\gamma=h-c$. In this work the associative hypernetted chain approximation (HNC) is used. In the absence of associative interactions it reduces to the $\mathrm{HNC}$ closure, this is used to evaluate the matrix structure

$$
c^{00}(r)=\left[1+f^{00}(r)\right] \exp \left[\gamma^{00}(r)\right]-1-\gamma^{00}(r)
$$

The HNC closures for fluid-matrix and fluid-fluid correlation functions are:

$$
c_{\alpha}^{10}(r)=\left[1+f^{10}(r)\right] \exp \left[\gamma_{0}^{00}(r)\right]\left[\delta_{\alpha, 0}+\delta_{\alpha, 1} \gamma_{1}^{10}(r)\right]-\delta_{\alpha, 0}-\gamma_{\alpha}^{10}(r)
$$

where $\delta_{\mu, \nu}$ is the Kronecker symbol, and

$$
c_{\alpha \beta}^{11}(r)=\left[1+f_{n o n}^{11}(r)\right] \exp \left[\gamma_{00}^{11}(r)\right]\left\{\delta_{\alpha, 0} \delta_{\beta, 0}+\delta_{\alpha, 1} \delta_{\beta, 0} \gamma_{\alpha \beta}^{11}(r)\right.
$$




$$
\left.+\delta_{\alpha, 1} \delta_{\beta, 1}\left[\left(\gamma_{10}^{11}(r)\right)^{2}+\gamma_{\alpha \beta}^{11}(r)\right]\right\}-\delta_{\alpha, 0} \delta_{\beta, 0}-\gamma_{\alpha \beta}^{11}(r)+\delta_{\alpha, 1} \delta_{\beta, 1} \exp \left[\gamma_{00}^{11}(r)\right] F_{a s}(r)
$$

However, in the case of ROZ equations, in addition to these closures, we also must use the closure for the blocking parts of the partial dcfs. The associative HNC closure for these functions reads

$$
\begin{aligned}
c_{\alpha \beta}^{11(2)}(r) & =\exp \left[\gamma_{00}^{11(2)}(r)\right]\left\{\delta_{\alpha, 0} \delta_{\beta, 0}+\delta_{\alpha, 1} \delta_{\beta, 0} \gamma_{\alpha \beta}^{11(2)}(r)\right. \\
& \left.+\delta_{\alpha, 1} \delta_{\beta, 1}\left[\left(\gamma_{10}^{11(2)}(r)\right)^{2}+\gamma_{\alpha \beta}^{11(2)}(r)\right]\right\}-\delta_{\alpha, 0} \delta_{\beta, 0}-\gamma_{\alpha \beta}^{11(2)}(r) .
\end{aligned}
$$

Finally the cavity distribution function in eq. (70) following from the associative HNC closure is

$$
y_{00}^{11}(r)=\exp \left\{h_{00}^{11}(r)-c_{00}^{11}(r)\right\} .
$$

Equations (63)-(65) together with (70)-(75) represent a complete associative ROZ-HNC problem for numerical solution. In previous studies of nonassociating and associating fluids in disordered porous media the Percus-Yevick closure also has been applied. It has been shown successful in many cases. Therefore, we also apply the associative Percus-Yevick closure for the system in question, however, only for the fluid-fluid and fluid-matrix correlations, because matrix structure is irrelevant. The associative Percus-Yevick closure reads

$$
\begin{aligned}
& c_{\alpha}^{10}(r)=f^{10}(r) y_{\alpha}^{10}(r), \\
& c_{\alpha \beta}^{11}(r)=f_{n o n}^{11}(r) y_{\alpha \beta}^{11}(r)+\left(1-\delta_{\alpha, 0}\right)\left(1-\delta_{\beta, 0}\right) y_{00}^{11}(r) F_{a s}(r), \\
& c_{\alpha \beta}^{11(2)}(r)=0,
\end{aligned}
$$

where

$$
\beta \mu_{e x}^{1}\left(\rho^{1}, \rho^{0}\right)=\beta \mu_{e x}^{1(h s)}\left(\rho^{1}, \rho^{0}\right)+\beta \mu_{e x}^{1(a s)}\left(\rho^{1}, \rho^{0}\right),
$$

$$
\begin{aligned}
& y_{\alpha}^{10}(r)=\delta_{\alpha 0}+h_{\alpha}^{10}(r)-c_{\alpha}^{10}(r) \\
& y_{\alpha \beta}^{11}(r)=\delta_{\alpha 0} \delta_{\beta 0}+h_{\alpha \beta}^{11}(r)-c_{\alpha \beta}^{11}(r) .
\end{aligned}
$$

The problem, either with the HNC or with the PY closure, has been straightforwardly solved numerically by direct iterations, only for high fluid densities one needs to take care about the convergency of the numerical scheme. In particular, to describe the states close to complete dimerization the association energy has been increased gradually to avoid the problems of convergency.

However, the structural properties which straightforwardly follow from the partial pair correlation functions and from eqs. (68) and (69) is not the only objective of our study. We intend to obtain thermodynamic properties as well. The easiest between them to obtain is the isothermal compressibility [20-22]. However, a challenge for the theory represent the adsorption isotherms, i.e. the $\beta \mu^{1}\left(\rho^{1}\right)$ dependencies.

We apply the calculation algorithm similar to that proposed by Ford and Glandt [22] for nonassociating fluids for the reference system which comprises a hard sphere fluid in a hard sphere matrix. The excess term is represented as a sum of two contributions where the first term corresponds to a reference system of hard spheres adsorbed in a disordered hard sphere matrix. It is obtained as discussed above.

The associative contribution into the chemical potential, $\beta \mu_{e x}^{1(a s)}\left(\rho^{1}, \rho^{0}\right)$, is taken from the result of thermodynamic perturbation theory applied to a pure associating fluid at the density $\rho^{1}[64]$

$$
\begin{aligned}
\beta \mu_{e x}^{1(a s)}\left(\rho^{1}\right) & =\ln \chi-\frac{1}{2}(\chi-1) \\
& +\rho^{1}\left(\frac{\partial \chi}{\partial \rho^{1}}\right)_{\beta \varepsilon_{a s}}(1 / \chi-1 / 2),
\end{aligned}
$$

where $\chi$ is the fraction of unbounded fluid particles. However, the effect of the matrix species is taken into account in this expression by using $\chi\left(\rho^{1}, \rho^{0}\right)$ and $\left(\partial \chi / \partial \rho^{1}\right)$ from the present solution of the ROZ-HNC equations for associating fluid adsorbed in a disordered matrix. One would expect a higher accuracy of adsorption isotherms obtained from eqs. (77), (78) for low and intermediate 
dimerization of adsorbed fluid. It is essential to mention that the linear 'charging' scheme is used only for the reference hard sphere fluid which was shown to work sufficiently well [22]. The method presented here for chemically associating fluids have been used by us already [43] and seems reasonable. An analysis of the results for adsorption isotherms following from presented equations is given in the following section. Most important is to investigate equilibria for associating fluids comparing their properties in the bulk and in a disordered matrix.

\section{Results}

We present here some of our results. Those are presented in figs. 9-11. We consider first a dimerizing fluid at association energy $\beta \varepsilon_{a s}=14$ and $\beta \varepsilon_{a s}=16$ adsorbed in a disordered matrix with $\rho^{0}=0.190986$, fig. 9 . With this choice of the values for association energy, we observe that the fraction of unbounded particles decreases with increasing chemical potential of adsorbed fluid such that for high values of $\beta \mu^{1}$ the fluid becomes to be highly dimerized, fig. 9b. From the adsorption isotherms presented in fig. 9a we conclude that both theoretical approaches yield qualitatively similar results. A discrepancy between the TPT treatment and eq. (27) is larger for a higher value of association energy $\left(\beta \varepsilon_{a s}=16\right)$. Moreover, with increasing chemical potential of adsorbed species, the difference between the results of both approaches slightly increases. We are convinced that the adsorption isotherms are qualitatively correct. However, it is difficult to evaluate their accuracy at quantitative level for the time being, due to the lack of computer simulation results. Grand canonical simulations for the adsorbed model associating fluids of this study are nontrivial. It follows from the results presented in fig. 9a that the adsorption of a fluid characterized by a higher association energy, $\beta \varepsilon_{a s}=16$, i.e. at a lower temperature, is higher in comparison to a fluid with $\beta \varepsilon_{a s}=14$, i.e. at a higher temperature. Similar trends in the behavior of adsorption isotherms with temperature, for a fluid with nonassociative attractive potential, have been observed in the computer simulation study of Vega et al. [65].

In fig. 10 we present the adsorption isotherms for a given fluid $\left(\beta \varepsilon_{a s}=14\right)$ in matrices of different density, $\rho^{0}=10^{-2} ; 0.190986$ and 0.3 . As expected, the density of adsorbed fluid at a given chemical potential is lower in a more dilute matrix, fig. 10a. On the other hand, at a given value of the fluid chemical potential, the fraction of monomers is higher in a denser matrix, fig. 10b.

It is of interest to perform a comparison of the structure of a dimerizing fluid at a given value of the chemical potential in different matrices. We have chosen a fluid with association energy, $\beta \varepsilon_{a s}=14$, and make comparison of its structure in terms of the distribution functions (in the ROZ-HNC approximation), at the chemical potential $\beta \mu^{1}=1.0$ in two matrices, namely with the density $\rho^{0}=10^{-2}$ and $\rho^{0}=0.3$ (fig. 11).
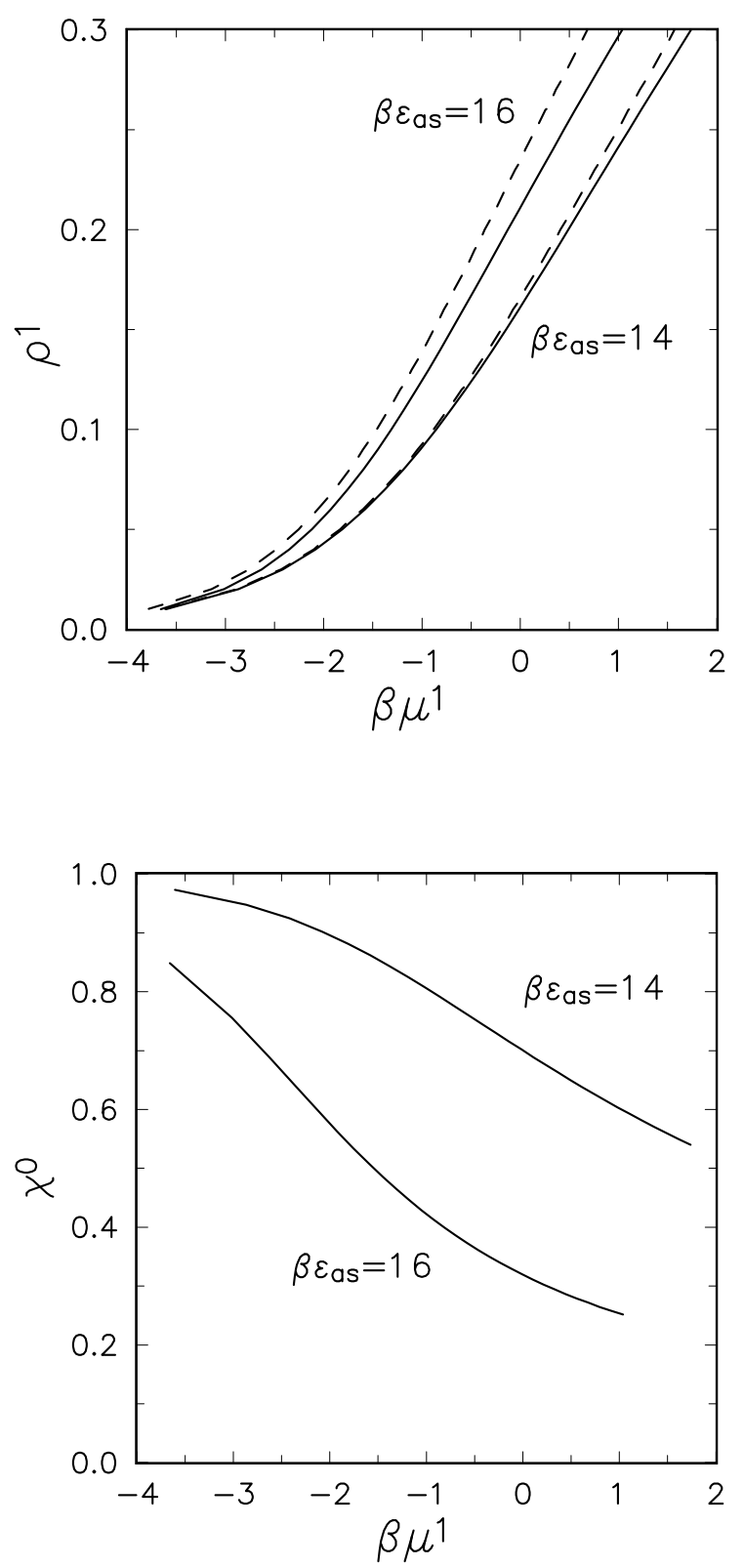

Fig. 9. The adsorption isotherms (part a) for a dimerizing fluid at $\beta \varepsilon_{a s}=14$ and at $\beta \varepsilon_{a s}=16$ in a disordered hard sphere matrix with density $\rho^{0}=0.190986$ obtained using the TPT estimate for the associative contribution (dashed lines), and using the connected part of the total direct correlation function (solid lines). The dependence of the fraction of monomers, $\chi^{0}$, for these adsorbed fluids is shown in part $b$.

We observe that the contact value of $g^{11}(r)$ is higher for the fluid adsorbed in a dense matrix, $\rho^{0}=0.3$ than in a dilute matrix; moreover the oscillations of $g^{11}(r)$ are stronger in the former case. It seems that the fluid behavior is due to the spatial restrictions for fluid particles arising in a dense matrix, in comparison with a dilute one. In the case of dilute matrix the blocking term of $g^{11}(r)$ is vanishing. The blocking contribution in the case $\rho^{0}=0.3$ is large in the intracore region. For larger distances it rapidly diminishes and does not play too 
serious role, as expected in absence of attractive fluidmatrix interactions, fig. $11 \mathrm{~b}$. The contact value for $g^{10}(r)$ is substantially higher for the case of dense matrix compared to the dilute one; trends for contact adsorption are stronger in a dense matrix, evidently. In both matrices, however, the fluid-matrix correlations decay fast as can be seen from fig. 11c.
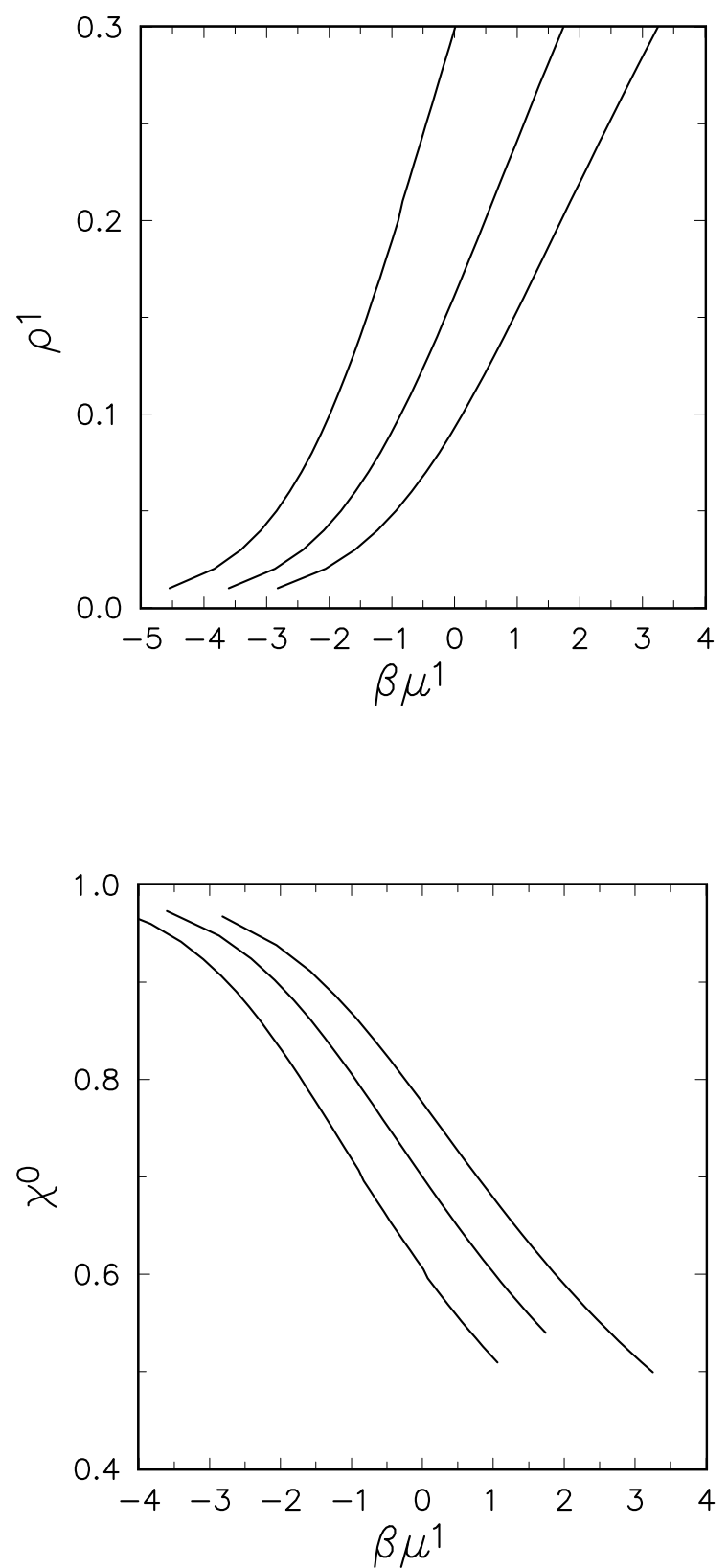

Fig. 10. The adsorption isotherms (part a) and the fraction of monomers for a dimerizing fluid (part b) at $\beta \varepsilon_{a s}=14$ in the matrices with the density $\rho=0.01,0.190986$ and 0.3 , from left to right in part a and from bottom to top in part $\mathrm{b}$, respectively. The curves have been obtained using the connected part of the total dcf with the ROZ-HNC approximation.
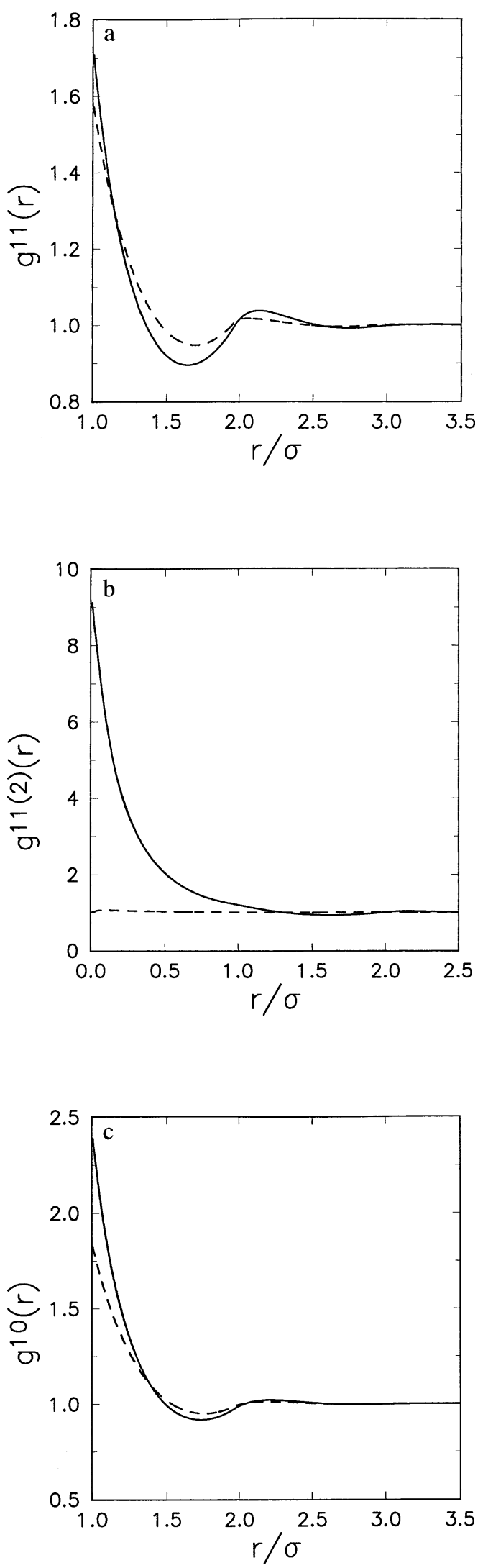

Fig. 11. A comparison of the structure of adsorbed dimerizing fluid at the chemical potential $\beta \mu^{1}=1.0$ in matrices with the density $\rho^{0}=0.01$ and $\rho^{0}=0.3$. The dashed and solid lines are for a lower and higher density matrix, respectively. The functions $g^{11}(r), g^{11(b)}(r), g^{10}(r)$ are given in parts a, b, and c, respectively. 
D. Application of the 4 site model of an associating fluid to study its adsorption in disordered porous media

Recently, we have investigated adsorption of the 4-site model for associating fluid in disordered hard sphere matrix using the associative ROZ equations [48]. The model is able to describe the formation of the network of bonds which is believed mimics the topology of the hydrogen bonding in water, in ammonia, in methanol, evidently, in a simplified manner. Moreover, the model intrinsically possesses liquid-vapor transition in contrast to the models of dimerization or chain formation. The model has been studied in the bulk conditions using thermodynamic perturbation theory and computer simulations [66-70], and near crystalline surface, in the framework of a simplified model of sticky sites [71]. We do not repeat theoretical background in detail referring to our original work [48] and aforementioned studies just cited. It is our principal focus to discuss the liquid-vapor coexistence curve of this model confined to disordered porous media. It has obtained according to the procedure discussed above, i.e. the chemical potential is evaluated via eqs. (75) and (76), then the Maxwell construction has been used for subcritical isotherms. The model has been solved using the associative PY approximation supplemented by the ideal network approximation [70, 71]. The coexistence curve of the 4-site fluid model adsorbed in a hard sphere matrices is presented in fig. 12. Note that the fluid and matrix particles are assumed to have the same diameter.

It is important to mention that the critical temperature becomes lower for adsorbed fluid w.r.t. the bulk for few percent, even for sufficiently dense matrices. The critical density is seriously influenced by the presence of the matrix. The critical density decreases. Moreover, the coexistence envelope shrinks, if the matrix density increases. Some of these features have been observed in the experimental studies, however, for fluids with nonassociative attraction. We are not aware of experiments and computer experiments for associating fluids adsorbed in disordered matrices, unfortunately. It is of interest to discuss the structure factor of adsorbed 4-site associative model. Particularly, a prepeak in the structure factor observed at small wave vectors is often attributed to the network formation. We present the structure factors of adsorbed fluid at $\beta \mu_{f}=-2.5$ in fig. 13. The region of intermediate fluid densities, at chosen value of the chemical potential describes adsorption of the model at $\beta \varepsilon_{a s}=12$ in matrices with $\rho_{m}=0.3 \div 0.4$. For these conditions the structure factor is characterized by the presence of the prepeak at small wave vectors. The height of the prepeak is lower for the fluid (at fixed chemical potential) in a denser matrices. In a high density matrix adsorbed density is lower, and for the wave vectors that have corresponded in the previous case to the prepeak, one observes a minimum of the structure factor. A more detailed discussion of this and related issues under the conditions of fixed chemical potential of adsorbed fluid one can find in Ref. [48]. Our expectation is that the model with four attractive sites is of interest to investigate further in combination with the model for adsorption of fluids in xerosilica gel of Kaminsky and Monson $[53]$.

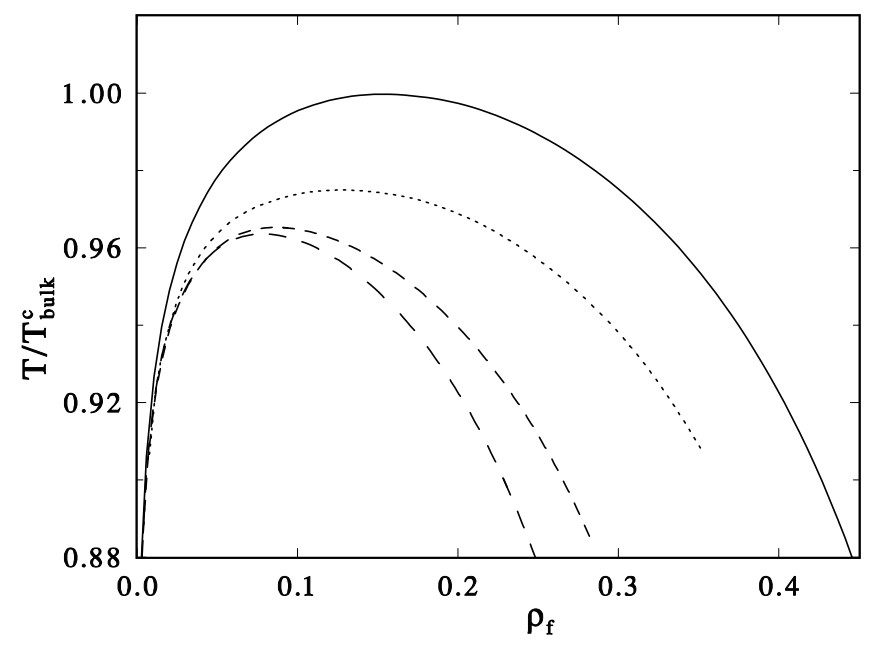

Fig. 12. Portions of the ROZ-PY coexistence envelopes, $T / T_{b u l k}^{c}$ versus fluid density, for the 4 -site associating fluid in a disordered hard sphere matrix at density $\rho_{m}=0$ (solid line), $\rho_{m}=0.1$ (dotted line), $\rho_{m}=0.3$ (dashed line), $\rho_{m}=0.45$ (long-dashed line). Temperature corresponds to $1 / \beta \varepsilon_{a s}$.

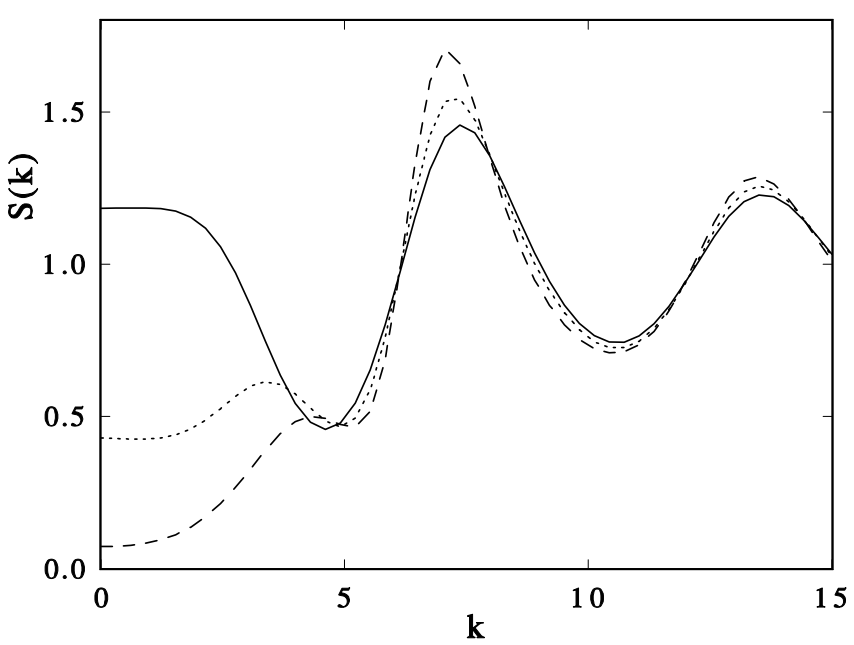

Fig. 13. The structure factor of adsorbed fluid with $\beta \varepsilon_{a s}=12$ at the chemical potential $\beta \mu^{f}=-2.5$. The fluid is adsorbed in disordered matrix at $\rho_{m}=0.1,0.3$, and 0.45 (dashed, short-dashed and solid lines, respectively). The curves follow from ROZ-PY approximation. 


\section{THE ROZ THEORY FOR PARTLY QUENCHED SYSTEMS WITH ELECTROSTATIC INTERACTIONS}

\section{A. General considerations}

It is of special interest for many applications to consider adsorption of fluids in matrices in the framework of models which include electrostatic forces. These systems are relevant, for example, to colloidal chemistry. On the other hand, electrodes made of specially treated carbon particles and impregnated by electrolyte solutions are very promising for practical applications. Only few attempts has been undertaken to solve models with electrostatic forces, those have been restricted, however, to Coulomb interactions. We would like to mention in advance, that it is clear, at present, how to obtain the structural properties of ionic fluids adsorbed in disordered charged matrices. Thermodynamics of these systems, and, in particular, peculiarities of phase transitions, is the issue which is practically unsolved, in spite of great importance. This part of our review is based on our recent works $[52,53]$.

It is well-known that to solve numerically integral equations for models with Coulomb interaction is difficult $[72,73]$. One needs to develop a renormalization scheme for the long-range terms of ion-ion correlations. Here we must do that for ROZ equations.

We consider a partly quenched mixture consisting of two ionic fluids of point-like particles. Each of the fluids contains a neutral combination of positively and negatively charged ions. One of the fluids describes a quenched charged system. It is equilibrated at temperature $T_{0}$ in a medium with dielectric constant $\varepsilon_{0}$. The quenched and fluid components are denoted by superscripts 0 and 1, respectively. An ionic fluid adsorbed in this quenched matrix is investigated at temperature $T_{1}$ in a medium with dielectric constant $\varepsilon_{1}$. Depending on the conditions of matrix preparation and on the conditions of observation, the parameter $\varepsilon_{1} T_{1}$ can be either smaller than $\varepsilon_{0} T_{0}$, equal to, or larger than $\varepsilon_{0} T_{0}$.

The charges of matrix ions are $e z_{+}^{0}=\left|e z_{-}^{0}\right|=e z^{0}$; and the density of the matrix subsystem is $\rho^{0}\left(\rho_{+}^{0}=\right.$ $\left.\rho_{-}^{0}=\rho^{0} / 2\right)$. We define the functions $\Phi_{i j}^{m n}(r)$ describing the interactions between particles. In particular, the interactions between matrix ions are given as

$$
\Phi_{i j}^{00}(r)=-U_{i j}^{00}(r) / \varepsilon_{0} k T_{0}=-e^{2} z_{i}^{0} z_{j}^{0} / \varepsilon_{0} k T_{0} r
$$

where $i$ and $j$ take values ' + ' and ' - '.

The electrolyte solution is modelled as a twocomponent, electroneutral system of point ions with charges $e z_{+}^{1}=\left|e z_{-}^{1}\right|=e z^{1}$. The density of the fluid is $\rho^{1}\left(\rho_{+}^{1}=\rho_{-}^{1}=\rho^{1} / 2\right)$. The fluid-fluid and fluid-matrix Coulomb interactions are

$$
\Phi_{i j}^{11}(r)=-U_{i j}^{11}(r) / \varepsilon_{1} k T_{1}=-e^{2} z_{i}^{1} z_{j}^{1} / \varepsilon_{1} k T_{1} r
$$

and

$$
\Phi_{i j}^{10}(r)=-U_{i j}^{10}(r) / \varepsilon_{1} k T_{1}=-e^{2} z_{i}^{1} z_{j}^{0} / \varepsilon_{1} k T_{1} r
$$

In addition, it is convenient to define the Bjerrum length parameter $L_{b}=e^{2}\left(z^{1}\right)^{2} / \varepsilon_{r} \varepsilon_{1} k T_{1}$ (where $\varepsilon_{r}$ is the universal dielectric constant) and the so-called quenching parameter, defined as $Q=\varepsilon_{0} T_{0} / \varepsilon_{1} T_{1}$.

\section{B. Elements of theoretical procedure and some results}

The correlation functions of the partly quenched system satisfy a set of replica Ornstein-Zernike equations (21) $-(23)$. Each of them is $2 \times 2$ matrix equation for the model in question. Similarly to previous studies of ionic systems, see eg. [72, 73], we denote the long-range terms of the pair correlation functions in ROZ equations by $q_{i j}$. Here we apply a linearized theory and assume that the long-range terms of the direct correlation functions are equal to the Coulomb potentials $\Phi_{i j}$, which are given by eqs. (79)-(81). This assumption represents the mean spherical approximation for the model in question. Most importantly, $\Phi_{i j}^{12}(r)=0$; as mentioned before, the particles from different replicas do not interact. However, $q_{i j}^{12}(r) \neq 0$, these functions describe screening effects of the ion-ion interactions between ions from different replicas mediated by the presence of charged obstacles, i.e. via the matrix. The functions $q_{i j}^{12}(r)$ need to be obtained to apply them for proper renormalization of the ROZ equations for systems made of non-point ions.

The equation determining matrix structure is

$$
\mathbf{q}^{00}-\boldsymbol{\Phi}^{00}=\boldsymbol{\rho}^{0} \boldsymbol{\Phi}^{00} \otimes \mathbf{q}^{00}
$$

where $\rho^{0}$ is $2 \times 2$ diagonal matrix with diagonal elements $\rho_{+}^{0}=\rho_{-}^{0}=\rho_{0} / 2$. We next perform the calculations using the ROZ equations for the fluid-matrix and fluidfluid correlations. By restricting analysis to the symmetric case, i.e. to 1-1 electrolytes adsorbed in 1-1 charged matrix, we can simplify the problem, taking advantage of the symmetry of the correlation functions. The equations for $\mathbf{q}^{10}, \mathbf{q}^{11}$ and $\mathbf{q}^{12}$ read:

$$
\begin{aligned}
& \mathbf{q}^{10}-\boldsymbol{\Phi}^{10}=\boldsymbol{\rho}^{0} \boldsymbol{\Phi}^{10} \otimes \mathbf{q}^{00}+\boldsymbol{\rho}^{1} \boldsymbol{\Phi}^{11} \otimes \mathbf{q}^{10} \\
& \mathbf{q}^{11}-\boldsymbol{\Phi}^{11}=\boldsymbol{\rho}^{0} \boldsymbol{\Phi}^{10} \otimes \mathbf{q}^{10}+\boldsymbol{\rho}^{1} \boldsymbol{\Phi}^{11} \otimes \mathbf{q}^{11} \\
& \mathbf{q}^{12}=\boldsymbol{\rho}^{0} \boldsymbol{\Phi}^{10} \otimes \mathbf{q}^{10}+\boldsymbol{\rho}^{1} \boldsymbol{\Phi}^{11} \otimes \mathbf{q}^{12}
\end{aligned}
$$

The matrix $\rho^{1}$ is $2 \times 2$ diagonal matrix with diagonal elements $\rho_{+}^{1}=\rho_{-}^{1}=\rho_{1} / 2$. Equations (82) and (83) can be readily solved to obtain Fourier-transforms of the screened potentials.

The expressions for the screened potentials in Cartesian space are obtained then straightforwardly: 


$$
\begin{aligned}
& \left(\begin{array}{cc}
q_{++}^{00}(r) & q_{+-}^{00}(r) \\
q_{+-}^{00}(r) & q_{--}^{00}(r)
\end{array}\right)=L_{b} / Q\left(\begin{array}{cc}
-1 & 1 \\
1 & -1
\end{array}\right) \exp \left(-\kappa_{0} r\right) / r \\
& \left(\begin{array}{ll}
q_{++}^{10}(r) & q_{+-}^{10}(r) \\
q_{+-}^{10}(r) & q_{--}^{10}(r)
\end{array}\right)=\frac{1}{Q}\left[\kappa_{1}^{2} /\left(\kappa_{0}^{2}-\left(\kappa_{1}^{2}\right)\right] L_{b}\left(\frac{\rho^{0}}{\rho^{1}}\right)\left(\begin{array}{cc}
-1 & 1 \\
1 & -1
\end{array}\right)\left[\exp \left(-\kappa_{0} r\right) / r-\left(\frac{\kappa_{1}^{2}}{\kappa_{0}^{2}}\right) \exp \left(-\kappa_{1} r\right) / r\right]\right.
\end{aligned}
$$

for the interactions between matrix ions and for the interactions between fluid and matrix ions, respectively. Here $\kappa_{0}=\left(4 \pi \rho^{0} L_{b} / Q\right)^{1 / 2}$ and $\kappa_{1}=\left(4 \pi \rho^{1} L_{b}\right)^{1 / 2}$ are the reciprocal Debye-Hückel radii of screening for the matrix and fluid ionic subsystem, respectively. For the correlations between fluid ions we have

$$
\left(\begin{array}{ll}
q_{++}^{11}(r) & q_{+-}^{11}(r) \\
q_{+-}^{11}(r) & q_{--}^{11}(r)
\end{array}\right)=L_{b}\left(\begin{array}{cc}
-1 & 1 \\
1 & -1
\end{array}\right) \exp \left(-\kappa_{1} r\right) / r+\left(\begin{array}{cc}
q_{++}^{12}(r) & q_{++-}^{12}(r) \\
q_{+-}^{12}(r) & q_{--}^{12}(r)
\end{array}\right)
$$

where the blocking contributions are the following

$$
\begin{aligned}
\left(\begin{array}{cc}
q_{++}^{12}(r) & q_{+-}^{12}(r) \\
q_{+-}^{12}(r) & q_{--}^{12}(r)
\end{array}\right) & =L_{b} Q\left[\kappa_{0}^{4} /\left(\kappa_{0}^{2}-\kappa_{1}^{2}\right)^{2}\right]\left\{\exp \left(-\kappa_{0} r\right) / r\right. \\
& \left.-\left[\exp \left(-\kappa_{1} r\right) / r\right]\left[1-\left(1-\kappa_{1}^{2} / \kappa_{0}^{2}\right) \kappa_{1} r / 2\right]\right\}\left(\begin{array}{cc}
-1 & 1 \\
1 & -1
\end{array}\right)
\end{aligned}
$$

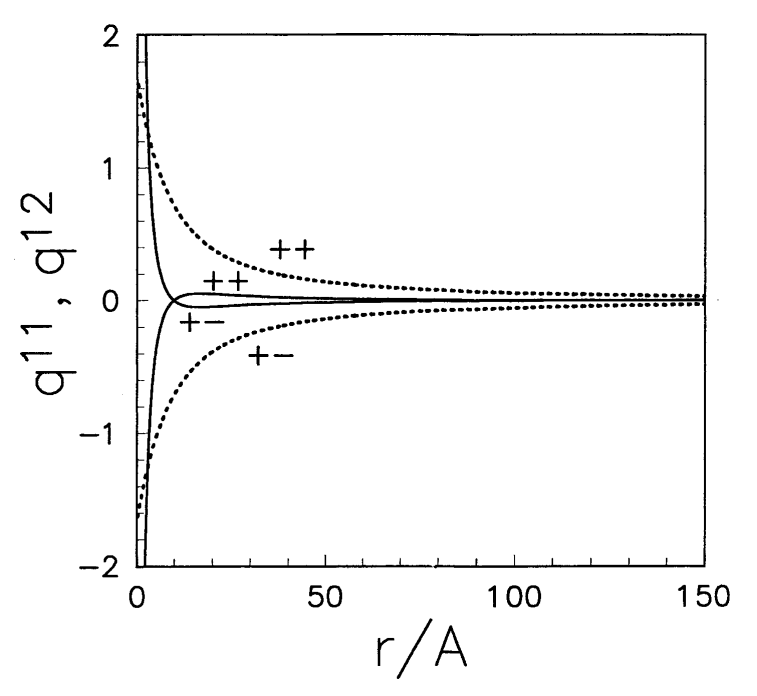

Fig. 14. The screened potentials of fluid ion-ion interactions, $q_{+-}^{11}(r)$ and $q_{++}^{11}(r)$ (solid lines) and blocking parts of the screened potentials, $q_{+-}^{12}(r)$ and $q_{++}^{12}(r)$ (dashed lines), at the matrix concentration $c^{0}=0.425 M$ and at the fluid concentration $c^{1}=6.8325 \times 10^{-5} M$.

It is evident that under certain conditions, unusual shape of $q_{i j}^{11}(r)$ functions may be expected. This is a result of the blocking term contribution. Some rearrangement of terms is necessary for the case where $\kappa_{1}=\kappa_{0}$, but no peculiar behavior is observed under these conditions. It is worth emphasizing that our result for $q_{i j}^{11}(r)$ generalizes the expression obtained by Bratko and Chakraborty for infinite dilution of fluid particles, see eq. (19) of Ref. [50]. The $q_{i j}^{11}(r)$ functions coincide with their result for $\rho^{1} \rightarrow 0$.

Let us discuss some of the results obtained so far. We present the screened potentials of Coulomb interaction between point ions in the quenched matrix of point-like ions in figs. 14 and 15. We also give the blocking contributions into the screened potentials in these figures. Let us comment the curves obtained. In fig. 14 we observe that at the matrix concentration $c^{0}=0.425 \mathrm{M}$ and at the fluid concentration $c^{1}=6.8325 \times 10^{-5} M$ for the system with quenching parameter, $Q=1.2$, the blocking term makes large effect on the behavior of the screened potentials of fluid ions. This effect yields that at small interparticle separations like-charged ions repel each other, however, at large separations net attraction is observed. The blocking terms are of much longer range than the screened potentials. The crossing point which can be seen in this figure lies approximately at the same distance as obtained Bratko and Chakraborty using computer simulations [50] for the same conditions.

In fig. 15 we show the results which demonstrate the effect of quenching parameter on the screening of interactions between fluid ions. Here two values of quenching parameter have been examined, $Q=0.7$ and $Q=1.7$ at a matrix concentration $c^{0}=1 M$ and at fluid concentration $c^{1}=0.75 \times 10^{-2} M$. It follows from the re- 
sults presented that at small interparticle distances the blocking effect is larger for $Q=1.7$ than for $Q=0.7$. Prequenching conditions provide that at $Q=1.7$ we observe two points of crossing of the screened potentials and no crossing points can be seen at $Q=0.7$. These results qualitatively coincide with computer simulation data [50]. With the screened potentials available it is straightforward to solve the problem of renormalization of the ROZ equations for any system of nonpoint ions.
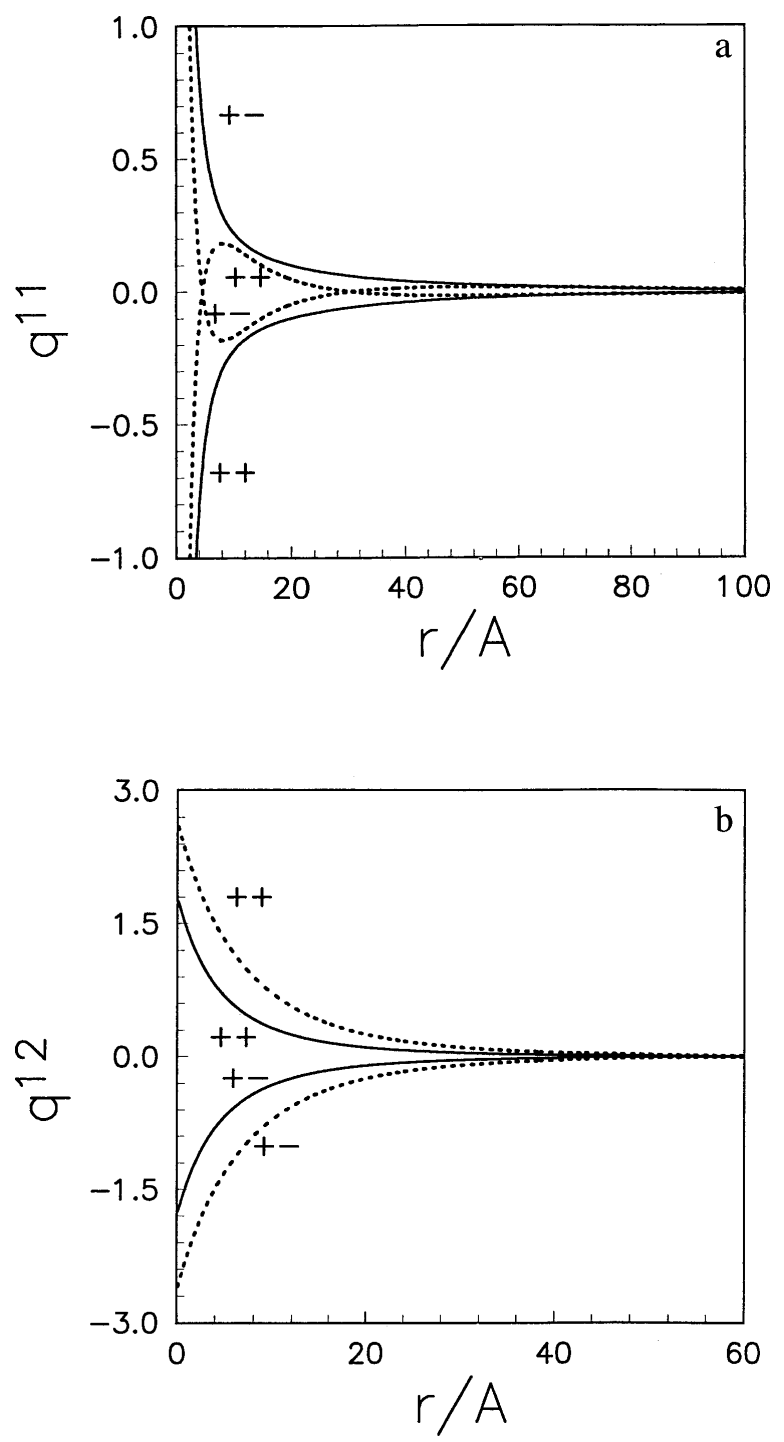

Fig. 15. The screened potentials of fluid ion-ion interactions, $q_{+-}^{11}(r)$ and $q_{++}^{11}(r)$ (part a) and blocking parts of the screened potentials, $q_{+-}^{12}(r)$ and $q_{++}^{12}(r)$ (part b) at different values of the quenching parameter. The solid and dashed lines correspond to $Q=0.7$ and $Q=1.7$, respectively.

\section{CONCLUSIONS}

In conclusion we have presented a review of some recent developments in the theory of partly quenched fluid systems. Our main focus has been on the applications of the replica Ornstein-Zernike equations for these systems. Several extensions have been discussed. Particularly we have considered recently developed inhomogeneous replica $\mathrm{OZ}$ equations and associative replica $\mathrm{OZ}$ equations. These approaches have been only pioneered and are quite far to be complete.

The structural properties of various systems are sufficiently straightforwardly obtained from integral equations. However, thermodynamics is much more difficult to obtain. Of particular importance, and far from being solved, are the issues related with phase transitions in partly quenched systems, even for simple models with attractive interactions. We would like to mention interesting possibilities which arise due to the inhomogeneous replica OZ equations to study phase transitions in pores, in membranes made of disordered and random matrices. On the other hand it would be of interest to answer the question concerning the influence of association between fluid particles and/or with matrix obstacles on the phase diagram of Lennard-.Jones fluid in model xerosilica gel and aerogels.

More sophisticated models, especially with long-range electrostatic forces await their turn to be studied. Phase transitions in Coulombic systems confined to disordered media is one of challenges for the theory. Charged colloids adsorbed in charged media represent one more interesting subject. On the other hand important applications may be expected from the relevant developments for dipolar confined fluids. Even without inclusion of long-range forces many models remain out of question. Very recently we have undertaken studies of quenchedannealed fluids in which matrix particles are permeable for fluid species [74]. There appear possibilities to involve modelling close to the models of biological membranes into the integral equations theory and computer simulations [75].

One can expect further progress not only along the line of studies involving integral equations. Computer simulations have already shown their ability to perform with success in this area of research. On the theoretical side we are not aware of the developments via a density functional theory for confined systems but Refs. [76, 77]. Of particular importance seems to be the methodology proposed in [77], because the problem of crystallization of fluids in disordered media is of principal interest and can be studied by these tools. Much future work is needed, however, to solve these problems.

\section{ACKNOWLEDGMENTS}

This project has been supported in parts by Silicon Graphics Inc. - Cray Research of Mexico under its University Research and Development grant, by DGAPA of the UNAM under research grants IN207796, IN111597, by CONACyT of Mexico under Grant 25301-E and by the KBN of Poland under grant No.3T 09A 06210. Majority of the results presented here have been obtained 
using the supercomputer facilities Origin 2000 of the supercomputer center of the UNAM.

We are grateful to our friends and collaborators, P. Bryk, Yu. Duda, D. Henderson, A. Huerta, Yu. Holovatch, B. Hribar, Ya. Ilnytskyj, G. Anguiano Orozco,
P. Padilla, A. Patrykiejew, G. Ponedilok, V. Renugopalakrishnan, A. Trokhymchuk, C. Vega, V. Vlachy for helpful discussions during preparation of the manuscript. We are especially indebted to P. Bryk and Yu. Duda for careful reading of the manuscript.
[1] M. Mezard, G. Parisi, M. A. Virasoro, Spin Glass Theory and Beyond (World Scientific, Singapore, 1987).

[2] I. A. Vakarchuk, Yu. K. Rudavskii, H. V. Ponedilok, Theor. Mat. Fiz. 58, 445 (1984).

[3] I. A. Vakarchuk, Yu. K. Rudavskii, H. V. Ponedilok, Phys. Status Solidi B 128, 231 (1985).

[4] I. A. Vakarchuk, I. F. Marholych, Phys. Status Solidi B 149, 301 (1988).

[5] I. A. Vakarchuk, V. M. Myhal, V. M. Tkachuk, Phys. Status Solidi B 166, 53 (1991).

[6] B. J. Frisken, A. J. Liu, D. S. Cannell, Mater. Res. Soc. Bull. May, 19 (1994).

[7] B. J. Frisken, D. S. Cannell, Phys. Rev. Lett. 69, 632 (1992).

[8] A. P. Y. Wong, M. H. W. Chan, Phys. Rev. Lett. 65, 2567 (1990).

[9] M. C. Goh, W. I. Goldburg, C. M. Knobler, Phys. Rev. Lett. 58, 1008 (1987)

[10] reported by F. Aliev at XXVII Winter Meeting on Statistical Physics, Cuernavaca, Mexico; unpublished.

[11] G. Viramontes-Gamboa, J. L. Arauz-Lara, M. MedinaNoyola, Phys. Rev. Lett. 75, 759 (1995).

[12] G. Viramontes-Gamboa, J. L. Arauz-Lara, M. MedinaNoyola, Phys. Rev. E 52, 4035 (1995).

[13] F. do Rosario, A. M. Travalloni Louvisse, S. M. Saraiva, G. Gonzales and J. F. de Oliveira, J. Coll. Interface Sci. 181, 1 (1996).

[14] W. G. Madden, E. D. Glandt, J. Stat. Phys. 51, 537 (1988).

[15] W. G. Madden, J. Chem. Phys. 96, 5422 (1988).

[16] J. A. Given, G. Stell, J. Chem. Phys. 97, 4573 (1992).

[17] J. A. Given, G. Stell, Physica A 209, 495 (1994).

[18] J. A. Given, G. Stell, in XVI International Workshop on Condensed Matter Theories, San Juan, Puerto Rico, 1992 (Plenum, New York, 1993).

[19] E. Lomba, J. A. Given, G. Stell, J. J. Weis, D. Levesque, Phys. Rev. E 48, 233 (1993).

[20] A. Meroni, D. Levesque, J. J. Weis, J. Chem. Phys. 105, 1101 (1996).

[21] M. L. Rosinberg, G. Tarjus, G. Stell, J. Chem. Phys. 100 5172 (1994).

[22] D. M. Ford, E. D. Glandt, J. Chem. Phys. 100, 2391 (1994).

[23] D. M. Ford, E. D. Glandt, Phys. Rev. E 50, 1280 (1994).

[24] J. A. Given, J. Chem. Phys. 102, 2934 (1995).

[25] E. Kierlik, M. L. Rosinberg, G. Tarjus, P. A. Monson, J. Chem. Phys. 103, 4256 (1995).

[26] E. Kierlik, M. L. Rosinberg, G. Tarjus, P. A. Monson, J. Chem. Phys. 106, 264 (1997).

[27] E. Pitard, M. L. Rosinberg, G. Stell, G. Tarjus, Phys. Rev. Lett. 74, 4361 (1995).

[28] S. Page, P. A. Monson, Phys. Rev. E 54, R29 (1996).
[29] P. A. Gordon, E. D. Glandt, J. Chem. Phys. 105, 4257 (1996).

[30] W. Dong, E. Kierlik, M. L. Rosinberg, Phys. Rev. E 50, $4750(1994)$.

[31] O. Pizio, S. Sokolowski, Phys. Rev. E 56, R63 (1997).

[32] A. Kovalenko, S. Sokolowski, D. Henderson, O. Pizio, Phys. Rev. E 58, 1824 (1998).

[33] Yu. Duda, P. Bryk, O. Pizio, S. Sokolowski, J. Phys. Chem. (submitted).

[34] C. Vega, P. Padilla, J. Chem. Phys. 106, 1997 (1997).

[35] A. Feng, B. J. McCoy, Z. A. Munir, D. E. Cagliostro, J. Coll. Interface Sci. 180, 276 (1996).

[36] J. Font, R. P. Castro, Y. Cohen, J. Coll. Interface Sci. 181, 347 (1996).

[37] M. S. Wertheim, J. Stat. Phys. 35, 19; 35 (1984).

[38] M. S. Wertheim, J. Stat. Phys. 42, 459; 477 (1985).

[39] Yu. V. Kalyuzhnyi, G. Stell, Mol. Phys. 78, 1247 (1993).

[40] D. Henderson, A. Patrykiejew, O. Pizio, S. Sokolowski, Physica A 233, 67 (1995).

[41] A. D. Trokhymchuk, O. Pizio, M. F. Holovko, S. Sokolowski, J. Phys. Chem. 100, 17004 (1996).

[42] A. D. Trokhymchuk, O. Pizio, M. F. Holovko, S. Sokolowski, J. Chem. Phys. 106, 200 (1997).

[43] G. Anguiano Orozco, O. Pizio, S. Sokolowski, A. D. Trokhymchuk, Mol. Phys. 91, 625 (1997).

[44] O. Pizio, A. Trokhymchuk, D. Henderson, S. Labik, J. Coll. Interface Sci. 191, 86 (1997).

[45] O. Pizio, Yu. Duda, A. Trokhymchuk, S. Sokolowski, J. Mol. Liq. (in press).

[46] P. Bryk, O. Pizio, S. Sokolowski, Mol. Phys. (in press).

[47] P. Padilla, O. Pizio, A. Trokhymchuk, C. Vega, J. Phys. Chem. 102, 3012 (1998).

[48] A. Kovalenko, O. Pizio, J. Chem. Phys. 108, 8651 (1998).

[49] D. Bratko, A. K. Chakraborty, Phys. Rev. E 51, 5805 (1995).

[50] D. Bratko, A. K. Chakraborty, J. Chem. Phys. 104, 7700 (1996).

[51] A. K. Chakraborty, D. Bratko, D. Chandler, J. Chem. Phys. 100, 1528 (1994).

[52] B. Hribar, O. Pizio, A. Trokhymchuk, V. Vlachy, J. Chem. Phys. 107, 6335 (1997).

[53] B. Hribar, O. Pizio, A. Trokhymchuk, V. Vlachy, J. Chem. Phys. 109, 2480 (1998).

[54] G. A. Mansoori, N. F. Carnahan, K. E. Starling, T. W. Leland, J. Chem. Phys. 54, 1523 (1971).

[55] R. D. Kaminsky, P. A. Monson, J. Chem. Phys. 95, 2936 (1996).

[56] Yu. Duda, D. Henderson, O. Pizio, D. T. Wasan, J. Phys. Chem. 102, 1562 (1998).

[57] Yu. Duda, D. Henderson, O. Pizio, D. T. Wasan, Mol. Phys. (in press). 
[58] S. Labik, A. Malijevski, P. Vonka, Mol. Phys. 56, 709 (1985).

[59] F. Lado, J. Comput. Phys. 8, 417 (1971).

[60] T. Boublik, Mol. Phys. 63, 685 (1988).

[61] Yu. Duda, L. Perez, O. Pizio, S. Sokolowski, (unpublished).

[62] M. S. Wertheim, J. Chem. Phys. 86, (1985) 2929.

[63] A. Trokhymchuk, O. Pizio, (unpublished).

[64] G. Jackson, W. G. Chapman, K. E. Gubbins, Mol. Phys. 65, 1; 1057 (1988).

[65] C. Vega, R. D. Kaminsky, P. A. Monson, J. Chem. Phys. 99, 3003 (1993).

[66] J. Kolafa, I. Nezbeda, Mol. Phys. 61, 161 (1987).

[67] I. Nezbeda, J. Kolafa, Yu. Kalyuzhnyi, Mol. Phys. 68, 143 (1989).

[68] I. Nezbeda, G. A. Iglesias-Silva, Mol. Phys. 69, 767 (1990).
[69] D. Ghonasgi, W. G. Chapman, Mol. Phys. 79, 291 (1993).

[70] E. Vakarin, Yu. Duda, M. F. Holovko, Mol. Phys. 90, 611 (1997).

[71] E. Vakarin, Yu. Duda, M. F. Holovko, J. Chem. Phys. 107, 5569 (1997).

[72] T. Ichije, A. D. J. Haymet, J. Chem. Phys. 93, 8954 (1990).

[73] V. Vlachy, T. Ichije, A. D. J. Haymet, J. Am. Chem. Soc. 113, 1077 (1991).

[74] S. Sokolowski, O. Pizio, Mol. Phys. (submitted).

[75] O. Pizio, V. Renugopalakrishnan, A. Trokhymchuk, J. Chem. Phys. (submitted).

[76] L. Pérez, S. Sokolowski, O. Pizio, J. Chem. Phys. 109, 1147 (1998).

[77] G. I. Menon, C. Dasgupta, Phys. Rev. Lett. 73, 1023 (1994).

\title{
ЗАСТОСУВАННЯ МЕТОДУ РЕПЛІК ДО ОПИСУ СТРУКТУРИ І ТЕРМОДИНАМІКИ ФЛ ЮЇДІВ У НЕВПОРЯДКОВАНИХ АДСОРБЕНТАХ
}

\author{
О. Пізіо ${ }^{1}$, С. Соколовскі ${ }^{2}$ \\ ${ }^{1}$ Iнститут хемї Національного Університету Мексики, \\ Койоакан, 04510, Мехіко, Федеральний округ, Мексика \\ ${ }^{2}$ Відділ моделпвання фізико-хемічних процесів, \\ Університет Мариї Кюрі-Склодовськой, 200-31, Люблін, Польща
}

\begin{abstract}
У цьому огляді розглянуто проблему опису заморожених-відпалених систем, тобто флюїдів у невпорядкованих матрицях із фіксованою конфігурацією частинок. Використано метод реплік у теорії рідин. Обговорено основи статистичної механіки цих систем. Викладено теоретичні результати і числові дані для структури, термодинаміки і фазових переходів у цих системах. Особливу увагу приділено оригінальним результатам авторів. Обговорено двовимірні моделі та хемічно реагуючі плини у мікропористих середовищах. Наприкінці розглянуто проблему опису адсорбції заряджених флюїдів у заряджених матрицях. У більшості випадків ми зосередились на інтегральних рівняннях типу репліки. Такожобговорено результати комп'ютерної симуляції цих систем. Коротко дискутується можливість розвитку теорії для нових ва жливих
\end{abstract} задач. 